\title{
Rare Disasters and Credit Market Puzzles
}

\author{
Peter Christoffersen, Du Du and Redouane Elkamhi
}

\author{
CREATES Research Paper 2013-45
}




\title{
Rare Disasters and Credit Market Puzzles*
}

\author{
Peter Christoffersen \\ $\mathrm{Du} \mathrm{Du}$ \\ Rotman School, \\ Hong Kong University \\ Redouane Elkamhi \\ CBS and CREATES \\ of Science and Technology \\ Rotman School, \\ University of Toronto
}

May 23, 2013

\begin{abstract}
We embed systematic default, procyclical recovery rates and habit persistence into a model with a slight possibility of a macroeconomic disaster of reasonable magnitude. We derive analytical solutions for defaultable bond prices and show that a single set of structural parameters calibrated to the real economy-and not to bond prices-can simultaneously explain several key empirical regularities in credit markets. Our model captures the empirical level and volatility of credit spreads, generates a flexible credit risk term structure, and provides a good fit to a century of observed spreads. The model also matches the widespread skewness in index options. Finally, our model reveals a nonlinear relationship between bond and option prices that depends on the state of the economy and that helps explain conflicting empirical evidence found in the literature.
\end{abstract}

JEL codes: C60, G12, G13

Keywords: Credit spreads, volatility, term structure, option skewness, stochastic recovery, consumption risk.

\footnotetext{
${ }^{*}$ We gratefully acknowledge financial support from the Bank of Canada, Hong Kong RGC Research Grant (HKUST 641208), and SSHRC. For helpful comments we thank David Bates, Ling Cen, Sudipto Dasgupta, Jan Ericsson, Xavier Gabaix, Peter MacKay, Tom McCurdy, Raunaq Pungaliya, Mark Seasholes, Ngoc-Khanh Tran (our WFA discussant) and seminar participants at HEC Montreal, McGill University, Queens University, University of Iowa, University of Toronto, as well as the CICF, INFORMS, and WFA conferences.
} 


\section{Introduction}

We set ourselves the following challenge: Within the framework of rare macro disasters-and using a single set of parameters calibrated to the real economy-can we explain key stylized facts in credit markets when restricting disasters to be of reasonable size? The empirical credit market regularities we investigate are: The relative high average level and volatility of investment grade credit spreads for short and medium-term bonds, the upward-sloping average term structure of investment grade credit spreads, the highly nonlinear time series dynamic in credit spreads, and the time-varying relationship between credit spreads and option skewness.

Our analysis shows that the answer is yes: The stylized facts in credit markets are captured in a rare-disaster model that incorporates habit persistence, systematic default and counter-cyclical loss rates. We show that all of these features are required in order to match the stylized facts in credit markets. In particular, we argue that habit-formation alone is not enough to match the level, term structure and time-series dynamic in credit spreads.

The parameters in the model are calibrated to macro consumption, historical default rates, and historical loss-given-default rates (referred to as loss rates hereafter). The required magnitude of consumption disaster in our model is reasonable at $15 \%$ in annual terms with a disaster probability of $2 \%$ per annum. It is important to note that a single set of parameters are capable of matching all the stylized facts that we pursue. It is also important to emphasize that the parameters are not calibrated to observed bond and option prices. While our focus is on credit markets, our model also implies a default-free interest rate of $1.4 \%$ per year, an equity premium of $5.9 \%$ and an equity market volatility of $17.6 \%$ per year on average. These are all close to empirically observed values and show that our model is capable of simultaneously matching empirical regularities across markets.

The calibrated model generates average Baa-Treasury spreads of 114 bps and average AaaTreasury spreads of 7 bps at the 5-year maturity. These levels are comparable to the observed CDS spreads for Baa and Aaa rated firms (107 bps and 18.5 bps), and they are lower than observed corporate bond spreads, because our model only accounts for credit risk, while other factors such as liquidity play an important role in corporate bond pricing. When focusing on Baa-Aaa spreads, the model generates an average of $107 \mathrm{bps}$ with a volatility of $40.7 \mathrm{bps}$. These numbers are close to the observed levels of $88.5 \mathrm{bps}$ and $37.4 \mathrm{bps}$, and they compare well with both traditional structural models and more recent models (e.g., Chen, Collin-Dufresne, and Goldstein, 2009; Chen, 2010; and Bhamra, Kuhn and Strebulaev, 2010).

Our model relates the default of investment grade bonds to macro disasters which in turn makes default a nonzero probability event in the continuous-time limit. In addition, default for investment

grade bonds coincides with states of high marginal utility because risk aversion and loss rate also peak around default events. These features help generate an empirically relevant spread for short maturities. For longer maturities, our model-implied term structure of credit spreads tends to 
flatten for investment grade firms which is consistent with the empirical evidence. This feature is difficult to generate in traditional structural models because the stationary default boundary and positive risk neutral cash-flow growth typically drive long-maturity credit spreads to zero. Our model can generate both upward and hump-shaped spread term structures, which are documented empirically in Jarrow, Lando, and Turnbull (1997).

Following Chen, Collin-Dufresne, and Goldstein (2009) we also investigate our model's implication for the time-series variation in Baa-Aaa spreads during the past century. The model matches the historical mean (129 bps vs 126 bps), the standard deviation ( 80.1 bps vs. 79.8 bps), the minimum (42.6 bps vs $34.0 \mathrm{bps}$ ), and the maximum (432 bps vs $510 \mathrm{bps}$ ), and it captures the time-series dynamics in spreads.

There is a growing literature linking options and credit markets. ${ }^{1}$ However, when regressing credit spreads on option skewness the coefficient is sometimes positive, sometimes negative, and sometimes insignificant (Cremers, Driessen, Maenhout, and Weinbaum, 2008). Our model shows that the relationship between credit spreads for investment grade bonds and index option skewness depends on the prevailing state of the economy and the relationship is not always positive. More specifically, we show that investment grade credit spreads are always countercyclical while option skewness is not. It is instead a non-monotonic function of the state of the economy. This insight explains why the effect of option skewness is not robustly estimated in credit spread regressions in the literature.

In our model credit spreads and option skewness are both endogenously determined through their exposure to economic disasters. They both rise as we increase the consumption jump severity until they reach their respective empirical levels at our calibrated consumption jump level of $15 \%$. We conclude that allowing for rare disasters is important for generating empirically relevant option skewness while simultaneously obtaining the required credit spread levels for investment grade bonds.

Accounting for rare disasters and time-varying risk aversion induces a high and time-varying risk-adjusted default intensity which induces considerable short-term credit spreads in our model. The jump magnitude in the pricing kernel controls the price of default risk in our framework. The jump magnitude generates a wedge in the default intensity between the risk neutral measure and the actual measure. We find that the default intensity ratio between the two measures is about 4 which is consistent with empirical studies (e.g., Berndt, Douglas, Duffie, Ferguson and Schranz, 2008). This ratio shows that our model is able to generate empirically relevant credit spreads while relying on a realistically calibrated pricing kernel.

Our paper also contributes to the literature by including habit formation into the class of credit risk models that enables closed-form valuation for defaultable bonds. The closed-form bond pricing expressions we provide are very useful in empirical work and they facilitate our understanding of

\footnotetext{
${ }^{1}$ See, among others, Cao, Yu and Zhong (2010), Zhang, Zhou, and Zhu (2009), Collin-Dufresne, Goldstein and Martin (2001), Wang, Zhou and Zhou (2011), Cremers, Driessen, and Maenhout (2008), and Carr and Wu (2011).
} 
how time-varying habit and loss rates drive credit spreads and bond risk premia.

Our paper is anchored in the disaster risk literature (Rietz, 1988; Barro, 2006). ${ }^{2}$ As in the influential paper by Gabaix (2012), we advocate disaster risk as a unifying solution for asset pricing puzzles. There are, however, many important differences between the setup in Gabaix (2012) and ours. First, while Gabaix (2012) investigates a variable disaster framework, we keep the physical default intensity constant as in the standard Rietz-Barro setup and model investors' time-varying risk aversion using habit persistence. Second, our study demonstrates that a reasonable 15\% drop in the consumption level, along with time-varying risk aversion, is sufficient to replicate credit, equity and options market regularities in our model. Third, we provide a more detailed focus on various stylized facts in credit markets related to the term structure, state dependences, and time series variation in spreads.

Our paper is also related to the literature separately explaining puzzles in equity, option and credit markets. In credit markets, Chen (2010) and Bhamra, Kuhn and Strebulaev (2010) rely on a long-run risk framework in a regime-switching economy and countercyclical loss rates to explain both credit spread and leverage puzzles. Gourio (2012) develops a production economy that can capture the level of credit spreads. We enhance the ability to explain credit market regularities over time and across maturities using similar levels of economic restrictions and degrees of freedom. Relying also on habit formation, Chen, Collin-Dufresne and Goldstein (2009), henceforth CCDG, advocate idiosyncratic risk and a countercyclical default boundary as solutions to credit spread puzzles. In our view, countercyclical default boundaries are difficult to verify empirically and of course play no role in explaining index option regularities. Furthermore, the ability of CCDG to generate short-term spreads as well as a sensible term structure is unknown. We develop a model that combines habit persistence and counter-cyclical loss rates and relates puzzles in different markets to macroeconomics disasters as the single source of uncertainty. Finally, our paper is related to the literature on equilibrium option pricing (Benzoni, Collin-Dufresne, and Goldstein, 2011; and Du, 2011) as well as the literature that strives to link pricing in option and credit markets (Cremers, Driessen and Maenhout, 2008).

The remainder of the paper is organized as follows. We introduce the economic framework in Section 2. Section 3 derives closed-form bond prices and premia for defaultable and defaultfree bonds. Section 4 contains an empirical assessment of the model on credit spreads. Section 5 explores the model further and considers the joint pricing of defaultable bonds and index options. Section 6 concludes.

\section{The Economic Framework}

In this section we first outline the pricing kernel used in Du (2011) who allows for consumption disasters and habit persistence. We then extend the model by developing a default intensity structure

\footnotetext{
${ }^{2}$ See also Wachter (2006, 2012), Gourio (2008), Barro (2009), and Chen, Joslin and Tran (2012) among others.
} 
for firms of different credit quality as well as a counter-cyclical process for the loss rate. Finally we calibrate the model to consumption and default data.

\subsection{Preferences and Pricing Kernel}

Following Campbell and Cochrane (1999) and Menzly, Santos and Veronesi (2004), we assume that the representative agent in the economy maximizes expected utility of the form

$$
E\left[\int_{0}^{\infty} e^{-\rho t} u\left(C_{t}, H_{t}\right) d t\right]=E\left[\int_{0}^{\infty} e^{-\rho t} \ln \left(C_{t}-H_{t}\right) d t\right]
$$

where $C_{t}$ denotes aggregate consumption, $H_{t}$ denotes habit level, and $\rho$ denotes the subjective time-discount rate. The instantaneous risk aversion, $\gamma_{t}$, is given by

$$
\gamma_{t} \equiv-\frac{C_{t} u_{c c}\left(C_{t}, H_{t}\right)}{u_{c}\left(C_{t}, H_{t}\right)}=\frac{C_{t}}{C_{t}-H_{t}}=\frac{1}{S_{t}}
$$

where $S_{t}$ denotes the procyclical surplus consumption ratio, which can be thought of as a proxy for the state of the economy.

Following Menzly, Santos and Veronesi (2004), we assume that $\gamma_{t}$ follows a mean reverting process, perfectly negatively correlated with innovations in log consumption, $c_{t}$, that is,

$$
d \gamma_{t}=k_{\gamma}\left(\bar{\gamma}-\gamma_{t}\right) d t-\alpha_{\gamma}\left(\gamma_{t}-\beta\right)\left(d c_{t}-E_{t}\left[d c_{t}\right]\right)
$$

where $\bar{\gamma}$ is the long-run average risk aversion, $k_{\gamma}$ controls the speed of mean reversion, $\alpha_{\gamma}>0$ captures the sensitivity of $\gamma_{t}$ to consumption innovations, and $\beta \geq 1$ sets a lower bound for $\gamma_{t}$.

Following Wachter (2006) we assume that log consumption is subject to a low-probability negative jump

$$
d c_{t} \equiv d \log \left(C_{t}\right)=\mu_{c} d t+\sigma d B_{t}+J_{c} d N_{t}
$$

where $B_{t}$ is a standard Brownian motion, and $N_{t}$ is a Poisson process with constant intensity $\lambda_{c}$ that captures the random arrival of economic disaster. The jump size in logs is denoted by $J_{c}<0$. Upon the occurrence of the $i$-th disaster at time $t_{i}, \log$ consumption jumps from $c\left(t_{i}\right)$ to $c\left(t_{i}\right)+J_{c}$. For simplicity, we assume $J_{c}$ is constant, but the model implications are largely unchanged when allowing for random jump sizes. Using Ito's lemma for jump-diffusions (e.g., Appendix F of Duffie, 2001), we get

$$
d C_{t} / C_{t}=\mu d t+\sigma d B_{t}+J_{C} d N_{t}
$$

where $J_{C} \equiv e^{J_{c}}-1$ and $\mu \equiv \mu_{c}+\frac{1}{2} \sigma^{2}$.

Substituting (3) into (2), the $\gamma_{t}$-process can now be rewritten as

$$
d \gamma_{t} / \gamma_{t}=\mu_{\gamma t} d t+\sigma_{\gamma t} d B_{t}+J_{\gamma t} d N_{t}, \text { with }
$$




$$
\mu_{\gamma t}=k_{\gamma} \frac{\bar{\gamma}-\gamma_{t}}{\gamma_{t}}+\alpha_{\gamma} \frac{\gamma_{t}-\beta}{\gamma_{t}} J_{c} \lambda_{c}, \quad \sigma_{\gamma t}=-\alpha_{\gamma} \frac{\gamma_{t}-\beta}{\gamma_{t}} \sigma, \quad J_{\gamma t} \equiv \frac{\gamma_{t}}{\gamma_{t-}}-1=-\alpha_{\gamma} \frac{\gamma_{t}-\beta}{\gamma_{t}} J_{c}
$$

where $\gamma_{t-}$ denotes the value of $\gamma$ an instant before the occurrence of a jump. A negative consumption innovation-whether driven by diffusion or a jump-leads to a positive innovation in $\gamma_{t}$. The magnitudes of $\sigma_{\gamma t}$ and $J_{\gamma t}$ are both increasing in $\gamma_{t}$, implying that the volatility of risk aversion is increasing in the level of risk aversion.

The pricing kernel $\Lambda_{t}$ in the economy is given by

$$
\Lambda_{t}=e^{-\rho t} u_{C}\left(C_{t}, H_{t}\right)=e^{-\rho t} \gamma_{t} / C_{t}
$$

We see that $\Lambda_{t}$ is determined partly by economic fundamentals captured by aggregate consumption, $C_{t}$, and partly by the representative agent's risk aversion $\gamma_{t}$, which can be viewed as market sentiment. Ito's lemma gives

$$
\begin{gathered}
d \Lambda_{t} / \Lambda_{t}=\mu_{\Lambda t} d t+\sigma_{\Lambda t} d B_{t}+J_{\Lambda t} d N_{t}, \text { with } \\
\mu_{\Lambda t}=-\rho-\mu+\sigma^{2}+\mu_{\gamma t}-\sigma \sigma_{\gamma t}, \quad \sigma_{\Lambda t}=\sigma_{\gamma t}-\sigma, \quad J_{\Lambda t} \equiv \frac{\Lambda_{t}}{\Lambda_{t-}}-1=e^{-J_{c}}\left(J_{\gamma t}+1\right)-1 .
\end{gathered}
$$

By combining (9) with (6), a negative consumption innovation, whether driven by diffusion or jump, leads to an amplified positive innovation in the pricing kernel through the $\gamma_{t}$ innovation. Following the literature, we refer to $-\sigma_{\Lambda t}>0$ as the price of diffusive risk, and $-J_{\Lambda t}<0$ as the price of jump risk. Note that $\left|\sigma_{\Lambda t}\right|$ and $\left|J_{\Lambda t}\right|$ are both increasing in $\gamma_{t}$, implying larger compensation per unit of risk for higher levels of risk aversion which in turn occur in bad economic states.

\subsection{Modeling Physical Default}

Following Jarrow, Lando, and Turnbull (1997), Duffie and Singleton (1999), Duffie and Lando (2001), and Gabaix (2012) and others, we assume that the representative agent has incomplete knowledge of the firm's fundamentals. For a given credit class, $j$, we assume that bond defaults are driven by a systematic component modeled by $N_{j t}^{\text {sys }}$, with intensity $\lambda_{j}^{\text {sys }}$ as well as an idiosyncratic component modeled by $N_{j t}^{i d i o}$, which has intensity $\lambda_{j}^{i d i o}$. We can thus write

$$
d N_{j t}\left(\lambda_{j}\right)=d N_{j t}^{s y s}\left(\lambda_{j}^{s y s}\right)+d N_{j t}^{i d i o}\left(\lambda_{j}^{i d i o}\right)
$$

where $\lambda_{j}=\lambda_{j}^{\text {sys }}+\lambda_{j}^{i d i o}$. Recall that the sum of two independent Poisson processes itself is Poisson.

When tying firm default to macro consumption, we decompose the disaster jump process in (4) into a component that triggers default captured by the $N_{j t}^{s y s}$ process in (10) and an orthogonal residual component that does not trigger default, defined by $N_{j t}^{\perp}$, with intensity $\lambda_{j}^{\perp}=\lambda_{c}-\lambda_{j}^{\text {sys }}$. This decomposition enables some systematic variation in default while ensuring that consumption 
shocks can occur without necessarily causing firms' to default. We thus have

$$
d N_{t}\left(\lambda_{c}\right)=d N_{j t}^{s y s}\left(\lambda_{j}^{s y s}\right)+d N_{j t}^{\perp}\left(\lambda_{j}^{\perp}\right)
$$

where $\lambda_{c}=\lambda_{j}^{\text {sys }}+\lambda_{j}^{\perp}$. Under this specification, the correlation of arrivals of economic disaster and default for credit class $j$ can be computed as

$$
\operatorname{Corr}\left(d N_{j t}, d N_{t}\right)=\frac{\operatorname{Cov}\left(d N_{j t}, d N_{j t}^{s y s}+d N_{j t}^{\perp}\right)}{\sqrt{\lambda_{j}} \sqrt{\lambda_{c}} d t}=\frac{\lambda_{j}^{s y s}}{\sqrt{\lambda_{j}} \sqrt{\lambda_{c}}}=\sqrt{\frac{\lambda_{j}}{\lambda_{c}}} \frac{\lambda_{j}^{s y s}}{\lambda_{j}} .
$$

In Section 5.2, we analyze how defaultable bond prices in our model are impacted when the ratio of systematic default intensity to total default intensity changes.

Empirically, Bakshi, Madan, and Zhang (2006) find that defaults for investment grade bonds are related to severe economic conditions which we capture via consumption jumps. Their finding is consistent with the observation that investment grade bonds default with lower probability than economic disasters strike, and we thus calibrate default intensities to be much below $1 \%$ for 5 -year Aaa and Baa bonds compared with a consumption jump intensity of $2 \%$.

In the so-called structural approach to corporate bond pricing default is modelled as the first time firm-value falls below a certain boundary. As the firm cash-flow process, like our consumption process, is specified exogenously, structural models need to first fit the implied firm-level dynamics to empirical observations. Taking into account the fitted firm-level dynamics, the structural approach has a comparable degree of freedom for pricing with our approach. Both approaches are subject to the restrictions that model-implied default rates and default losses need to match empirical sample moments from Moody's or Standard \& Poor's, for example. Huang and Huang (2012) have demonstrated that matching default rates and default loss imposes strict economic restrictions on the ability of traditional structural models to explain credit spreads.

Our default modeling approach has several advantages. It is tractably embedded into the habit formation framework and it delivers closed-form expressions for bond prices and premia, which facilitates the analysis of the default premium as studied by Driessen (2005) and Berndt, Douglas, Duffie, Ferguson and Schranz (2008). Finally, our consumption-based model framework allows us to jointly analyze credit spreads and option prices.

\subsection{Modeling Loss Rates}

We focus on zero-coupon defaultable bonds which pay a face value of 1 at expiration date contingent on not defaulting. To capture default risk, we need to account for bond losses due to defaults. We assume that bondholders recover $1-L_{t}$ of the bond face value upon bankruptcy, where $L_{t}$ denotes the loss rate at the time of default.

Many studies including Altman and Kishore (1996), suggest that default loss is time varying. In addition, Shleifer and Vishney (1992) and Acharya, Bharath and Srinivasan (2007) argue that 
asset sales of distressed firms suffer from large discounts if an entire industry or the economy as a whole experiences financial distress, which implies a countercyclical loss rate. In view of this evidence, we model the loss rate process as

$$
d L_{t}=k_{L}\left(\bar{L}-L_{t}\right) d t-\alpha_{L}\left(d c_{t}-E_{t}\left[d c_{t}\right]\right)
$$

where $\bar{L}$ is the long run average of the recovery rate, $k_{L}$ controls the speed of mean reversion, and $\alpha_{L}$ captures the sensitivity of $L_{t}$ to consumption innovations. Using the consumption process in (4), we can rewrite (13) as

$$
d L_{t}=k_{L}\left(\bar{L}-L_{t}\right) d t-\alpha_{L} \sigma d B_{t}-\alpha_{L} J_{c}\left(d N_{t}-\lambda_{c} d t\right) .
$$

Our parsimonious specification of the loss rate process imposes a tight restriction on the dependence of the loss rate on macro variables. Intuitively, a negative consumption innovation implies a worsening of macroeconomic conditions which, under a positive $\alpha_{L}$, raises the loss rate. Chen (2010) provides empirical evidence on the close relation between the bond recovery rate, $1-L_{t}$, and consumption growth. In the Appendix we show how the loss rate specification can be extended by allowing for an additional shock which may be only partially correlated with consumption.

\subsection{Model Calibration}

We now calibrate parameters related to the dynamic processes for consumption, preferences and default. It is crucial to note that no parameter is calibrated to option or bond prices.

Consider first the consumption process in (4) which we calibrate as follows

Table 1.A: Consumption Parameters

\begin{tabular}{ccccc}
\hline$\mu$ & $\sigma$ & $\lambda_{c}$ & $J_{c}$ & $J_{C}$ \\
0.02 & 0.02 & 0.02 & -0.1625 & -0.15 \\
\hline \hline
\end{tabular}

In the model, $\mu$ and $\sigma$ denote, respectively, the mean and the volatility of consumption growth conditional on no disaster. Since disasters are rare by nature, $\mu$ and $\sigma$ should be close to the corresponding consumption sample moments. Following numerous previous studies we set $\mu=\sigma=$ $2 \%$. Consistent with the rare disaster literature, we set the jump intensity, $\lambda_{c}=0.02$, implying that economic jumps on average strike once every 50 years.

Calibrating the jump magnitude is complicated by the fact that large consumption drops are often followed by a strong recovery. Gabaix (2012) follows Barro (2006), and Barro and Ursua (2008) in calibrating the disaster magnitude to over 30\%. Constantinides (2008), Donaldson and Mehra (2008), and Gourio (2008) have noted that it is difficult to reconcile a disaster magnitude of $30 \%$ with the historical record. Using a new panel data set on personal consumer expenditure for 24 countries covering more than 100 years, Nakamura, Steinsson, Barro, and Ursua (2011) document that a peak-to-trough drop in consumption is on average $30 \%$ but half of this decline is reversed in 
subsequent recovery. Motivated by their findings, we set $J_{C}=-15 \%$ to reflect the net impact of consumption disasters on asset prices. The log consumption jump, $J_{c}$, is then $-16.25 \%$.

Consider next the preference parameters in (2) and (7) which we calibrate as follows

Table 1.B: Preference Parameters

\begin{tabular}{ccccc}
\hline$k_{\gamma}$ & $\bar{\gamma}$ & $\beta$ & $\alpha_{\gamma}$ & $\rho$ \\
0.16 & 33.97 & 20 & 39.4 & 0.04 \\
\hline
\end{tabular}

We take the preference parameter values $k_{\gamma}$ and $\bar{\gamma}$ directly from Menzly, Santos and Veronesi (2004) who focus on equity pricing. As they do not model jumps, we scale down $\alpha_{\gamma}$ from their value of 79.39 to adjust for the impact of consumption jumps on the equity premium. We set $\alpha_{\gamma}$ so that our model implies an equity premium of 5.9\%. Simultaneously, and consistent with Pan (2002), the model generates a jump risk premium which on average accounts for $38.9 \%$ of the total equity premium.

Consider finally the calibration of loss and default

Table 1.C: Loss and Default Parameters

\begin{tabular}{ccccccc}
\hline $\bar{L}$ & $k_{L}$ & $\alpha_{L}$ & $\lambda_{\text {Aaa }}^{\text {sys }}$ & $\lambda_{\text {Baa }}^{\text {sys }}$ & $\lambda_{\text {Aaa }}^{\perp}$ & $\lambda_{\text {Baa }}^{\perp}$ \\
0.586 & 0.4 & 2.7 & $0.0214 \%$ & $0.365 \%$ & $1.98 \%$ & $1.635 \%$ \\
\hline \hline
\end{tabular}

Following Chen (2010), we use simulated method of moments to estimate the three parameters, $\bar{L}, k_{L}$, and $\alpha_{L}$, that govern the loss rate process in (14). The target moments are a mean loss of $58.6 \%$, with a volatility of $9.6 \%$, and a first order autocorrelation of $63.5 \%$ corresponding to the empirical moments from Moody's aggregate recovery rate series from 1982 to 2008. The model predicted moments are based on a large sample of 100,000 loss rates simulated according to (14) for 100 years.

Following Huang and Huang (2012) and CCDG, and consistent with empirical data on physical default available from Moody's for the period 1970-2008, we set the 5-year cumulative default probability for Aaa and Baa bonds to $0.107 \%$ and $1.824 \%$, respectively. The annualized default intensity for these two bonds are thus $\lambda_{A a a}=0.0214 \%$ and $\lambda_{B a a}=0.365 \%$. In our benchmark calibration we set $\lambda_{j}^{\text {idio }}=0$ so that the $\lambda_{A a a}^{\text {sys }}=\lambda_{\text {Aaa }}$ for highly rated bonds and $\lambda_{B a a}^{\text {sys }}=\lambda_{B a a}$ for lower rated bonds. In Section 5.1 we discuss the impact of allowing for nonzero $\lambda_{j}^{i d i o}$. For each rating class, we fix the orthogonal consumption jump intensity via $\lambda_{j}^{\perp}=\lambda_{c}-\lambda_{j}^{\text {sys }}$, so that $\lambda_{\text {Aaa }}^{\perp}=.02-.0002=.0198$ and $\lambda_{\text {Baa }}^{\perp}=.02-.00365=.01635$.

In order to provide intuition for the calibrated model, we plot in Figure 1 a 120 -year simulated sample path. The top panel shows the log consumption path that features a jump around year 30 and another around year 70 . Note that the consumption disasters in the graph are quite reasonable in magnitude. The second row of panels shows the simulated surplus ratio path, $S$, which indicates booms and recessions in the economy (left panel) and the countercyclical loss rate (right panel). The bottom row of panels shows the number of defaults per year in a population of 1,000 Aaa 
firms and 1,000 Baa firms created at the beginning of the simulation. In the bottom-left panel of Figure 1 we follow the benchmark calibration and assume that Aaa-defaults are purely systematic and triggered solely by consumption disasters. Since $\lambda_{\text {Aaa }}<<\lambda_{c}$, only a small fraction of the 1,000 Aaa firms default when consumption disaster strikes, which is realistic. Purely for illustration, we deviate from the benchmark calibration by assuming that $35 \%$ of Baa defaults are idiosyncratic, and the simulated defaults are plotted in the bottom-right panel of the same figure. In comparison, we see more Baa firms default upon consumption disasters than Aaa firms, and in addition Baa firms also default during periods with no consumption jumps.

As forcefully argued in Huang and Huang (2012) and CCDG, calibrating loss and default rates to physical dynamics imposes tight economic restrictions on the model. Our calibration strategy ensures that our model has a comparable degree of freedom to traditional credit risk models. Note in particular that we are conducting a purely out-of-sample exercise when we apply the model for bond pricing below.

\section{Bond Prices and Risk Premia}

Above we have developed a 2-regime (default and no-default), 3-factor model with dynamics in consumption, habit, and loss rates. In this section we first derive expressions for the prices and premia of default-free and for defaultable bonds. We then provide some intuition for the model's ability to generate large credit spreads for investment grade firms. The proofs of all propositions

are provided in the Appendix. For brevity we suppress the dependence of prices on credit class in this section.

\subsection{Default-Free Bonds}

Proposition 1 The price of a zero-coupon default-free bond with $\tau$ years to expiration is given by

$$
P_{t, \tau}^{0}=\alpha_{1}^{0}(\tau)+\alpha_{2}^{0}(\tau) \tau \frac{1}{\gamma_{t}}
$$

where $\alpha_{1}^{0}(\tau)$ and $\alpha_{2}^{0}(\tau)$ are all positive. The instantaneous return on a default-free bond follows:

$$
\frac{d P_{t, \tau}^{0}}{P_{t, \tau}^{0}}=\mu_{P t}^{0} d t+\sigma_{P t}^{0} d B_{t}+J_{P t}^{0} d N_{t},
$$

with

$$
\begin{aligned}
\sigma_{P t}^{0} & =-\alpha_{2}^{0}(\tau) \frac{1}{\gamma_{t} \cdot P_{t, \tau}^{0}} \sigma_{\gamma t}, \\
J_{P t}^{0} & \equiv \frac{P_{t, \tau}^{0}}{P_{t-, \tau}^{0}}-1=\alpha_{2}^{0}(\tau) \frac{1}{\gamma_{t-} \cdot P_{t-, \tau}^{0}}\left(\frac{1}{1+J_{\gamma t}}-1\right)
\end{aligned}
$$


where $\sigma_{\gamma t}=-\alpha_{\gamma} \frac{\gamma_{t}-\beta}{\gamma_{t}} \sigma$ is the volatility of $\gamma_{t}$, and where $J_{\gamma t}=-\alpha_{\gamma} \frac{\gamma_{t}-\beta}{\gamma_{t}} J_{c}$ is the jump size of $\gamma_{t}$. The expression for $\mu_{P t}^{0}$ is provided in the Appendix.

We have verified that $\alpha_{2}^{0}(\tau)>0$, so that equation (15) shows that $P_{t, \tau}^{0}$ loads negatively on risk aversion $\gamma_{t}$ and hence positively on the surplus $S_{t}$. By combining the bond return process (16) with the pricing kernel process (8), the implied default-free bond risk premium is

$$
B P_{t}^{0}=-\sigma_{\Lambda t} \sigma_{P t}^{0}-\lambda_{c} J_{\Lambda t} J_{P t}^{0}
$$

where $\sigma_{\Lambda t}$ is the diffusive volatility and $J_{\Lambda}$ is the jump size of the pricing kernel given in (9). In (19), the two terms are compensations for diffusive and jump risks, respectively. As discussed in Wachter (2006), real default-free bonds are risky under habit formation. Their yields are low for high consumption surplus which generates an upward-sloping yield curve. This relation implies a negative covariance between dynamics of bond returns and the pricing kernel, and hence a positive bond risk premium.

It is straightforward to verify that when $\tau \rightarrow 0$, the implied bond yield $y_{t, \tau}^{0}=-\frac{1}{\tau} \ln P_{t, \tau}^{0}$ approaches the short term interest rate $r_{t}$ which by definition equals

$$
r_{t}=-E_{t}\left(d \Lambda_{t} / \Lambda_{t}\right)=-\mu_{\Lambda t}-\lambda_{c} J_{\Lambda t}
$$

where $\mu_{\Lambda t}$ and $J_{\Lambda t}$ are the drift and jump size of the pricing kernel given in (9). The risk-free rate is on average $1.4 \%$ per year using our parameter calibration.

\subsection{Defaultable Bonds}

Let $P_{t, \tau}$ be the time $t$ price of a zero-coupon defaultable bond, which has not yet defaulted, and which has $\tau$ years remaining until expiration. In the following we assume that in case of default the bond holder recovers face value (RFV). Given the pricing kernel of (8), we can prove the following proposition.

Proposition 2 Under the recovery of face value ( $R F V$ ) assumption, the price of a defaultable bond, which has not yet defaulted, is

$$
P_{t, \tau}=\alpha_{1}(\tau)+\alpha_{2}(\tau) \frac{1}{\gamma_{t}}+\alpha_{3}(\tau) L_{t}+\alpha_{4}(\tau) \frac{L_{t}}{\gamma_{t}}
$$

where the $\alpha_{i}(\tau)$ s are given in the Appendix.

We have verified that $\alpha_{1}(\tau), \alpha_{2}(\tau)$, and $\alpha_{4}(\tau)$ are positive, while $\alpha_{3}(\tau)$ and $\alpha_{3}(\tau)+\alpha_{4}(\tau) \frac{1}{\gamma_{t}}$ are negative. From (2), $\gamma_{t}$ has a lower bound of $\beta$ which bounds the magnitude of the positive term,

$\alpha_{4}(\tau) \frac{1}{\gamma_{t}}$. As a result, the $\alpha_{3}(\tau)$ term always dominates leading to a negative $\alpha_{3}(\tau)+\alpha_{4}(\tau) \frac{1}{\gamma_{t}}$ 
coefficient on $L_{t}$. Therefore, (20) says that a high risk aversion and a high loss rate both translate into a low defaultable bond price which is intuitive.

The next proposition summarizes the dynamics of $P_{t, \tau}$.

Proposition 3 The instantaneous return on an RFV defaultable bond conditional on no-default is given by

$$
\frac{d P_{t, \tau}}{P_{t, \tau}}=\mu_{P t} d t+\sigma_{P t} d B_{t}+J_{P t} d N_{t}^{\perp}+\left[\frac{1-L_{t}}{P_{t-, \tau}}-1\right] d N_{t}^{s y s}
$$

where the Poisson process $d N_{t}^{\text {sys }}$ denotes the arrival of default, and the orthogonal $d N_{t}^{\perp}$ process denotes arrivals of economic disasters that do not induce default. Furthermore, we have

$$
\begin{aligned}
\sigma_{P t} & =-\alpha_{2}(\tau) \frac{\sigma_{\gamma}}{\gamma_{t} P_{t, \tau}}-\alpha_{3}(\tau) \frac{\alpha_{L} \sigma}{P_{t, \tau}}-\alpha_{4}(\tau) \frac{L_{t} \sigma_{\gamma}+\alpha_{L} \sigma}{\gamma_{t} P_{t, \tau}} \\
J_{P t} & \equiv \frac{P_{t, \tau}}{P_{t-, \tau}}-1 \\
& =\alpha_{2}(\tau) \frac{1}{\gamma_{t-} P_{t-, \tau}}\left(\frac{-J_{\gamma}}{1+J_{\gamma}}\right)-\alpha_{3}(\tau) \frac{\alpha_{L} J_{c}}{P_{t-, \tau}}+\alpha_{4}(\tau) \frac{1}{\gamma_{t-} P_{t-, \tau}}\left[L_{t-}\left(\frac{-J_{\gamma}}{1+J_{\gamma}}\right)-\frac{\alpha_{L} J_{c}}{1+J_{\gamma}}\right],
\end{aligned}
$$

where $\sigma_{\gamma}$ and $J_{\gamma}$ are the diffusion and jump size of $\gamma_{t}$ from (6), and where $\alpha_{L}$ captures the sensitivity of $L_{t}$ to consumption innovations which in turn have volatility $\sigma$ and jump size $J_{c}$. The expression for $\mu_{P t}$ is provided in the Appendix.

To understand (21), note that the orthogonal decomposition of $d N_{t}^{\perp}$ and $d N_{t}^{\text {sys }}$ reflects the fact that the bond price jump size not triggered by default, denoted by $J_{P t}$, differs from the jump size

triggered by default, which equals $\frac{1-L_{t}}{P_{t-, \tau}}-1$. In the latter case, we have relied on the fact that under the RFV arrangement, upon default, the bond price immediately jumps to the recovery rate $1-L_{t}$.

Combining (21) with (8), we obtain the following risk premium demanded when holding the defaultable bond

$$
B P_{t}=-\sigma_{\Lambda t} \sigma_{P t}-\lambda^{\perp} J_{\Lambda t} J_{P t}-\left(\lambda^{s y s}+\lambda^{i d i o}\right) J_{\Lambda t}\left[\frac{1-L_{t}}{P_{t-, \tau}}-1\right] .
$$

In (22), the three terms are compensations for diffusive risk, jump risk not accompanied by default, and default risk, respectively.

\subsection{Model-Based Credit Spreads and Premia}

Given the price of defaultable bonds conditional on no-default, $P_{t, \tau}$, the model-implied yield is computed from $y_{t, \tau}=-\frac{1}{\tau} \ln \left(P_{t, \tau}\right)$. The model-based credit spread can then be computed as $C S_{t, \tau} \equiv y_{t, \tau}-y_{t, \tau}^{0}$, where we use the default-free bond as the benchmark. By using the physical default intensities, $\lambda_{A a a}$ and $\lambda_{B a a}$, calibrated in Table 1.C above, we can compute model-implied credit spreads for Aaa and Baa rated bonds. 
Figure 2 plots the model-implied Baa-Treasury and Aaa-Treasury spreads from the model. In order to investigate how our model generates credit spreads, we consider the following special cases: In the "Constant Loss and Habit" special case we set $k_{\gamma}=\alpha_{\gamma}=k_{L}=\alpha_{L}=0$. In this model, there is a positive relationship between the pricing kernel and default, but we have shut down dynamics in the loss rate and risk aversion. In the "Constant Habit" special case we allow for only variations in $L_{t}$ by setting $k_{\gamma}=\alpha_{\gamma}=0$. In the "Constant Loss Rate" case we allow for only variations in $\gamma$ by setting $k_{L}=\alpha_{L}=0$. The most general model where $\gamma$ and $L$ are both dynamic is labelled "Full Model" in Figure 2.

The top panel in Figure 2 illustrates the credit spread for a Baa bond as a function of $S=1 / \gamma$ when default loss $L_{t}$ is set to its unconditional value, $\bar{L}$. From Figure 2, the credit spreads are very low in the "Constant Loss and Habit" case which can be viewed as one of the risk-neutral structural models calibrated in Huang and Huang (2012). Allowing for variation in default loss in the "Constant Habit" model almost doubles the implied credit spreads. Note of course that when habit is constant the implied spreads do not react to variations in the surplus ratio. Allowing for habit formation only in the "Constant Loss Rate" case also adds substantially to the credit spread which now clearly exhibits counter-cyclicality. Finally, the full model further drives up model spreads to much more realistic levels. Perhaps the most striking result from Figure 2 is that the dynamic $L$ and $\gamma$ combine to have such a large effect in driving up the model credit spreads, as illustrated by the "Full Model" line.

The bottom panel of Figure 2 show the same special cases of our model but now for Aaa rated bonds, which reveals similar patterns across models. Specifically, credit spreads in our full model are countercyclical and reach realistically levels ranging from 5 to $18 \mathrm{bps}$.

In Figure 3 we again plot the Baa-Treasury credit spread (solid line in top panel) and the Aaa-Treasury credit spread (solid line in bottom panel), but we now focus on the full model and plot within the same figure the bond premium differences $B P_{t}-B P_{t}^{0}$ (dashed lines). While credit spreads are counter-cyclical for all values of the surplus ratio, bond premium differences are procyclical for extremely low levels of the surplus ratio and countercyclical for low, moderate and high levels of surplus. Due to the nonlinearities in the model, the premium differences exceed the credit spread differences for certain values of $S$.

In external habit models, the volatility of $S$, which we can think of as systemic risk, vanishes as $S$ goes to zero so as to prevent negative marginal utility. This decrease in systematic risk as $S$ goes to zero results in a decrease in bond return volatility and bond price jump sizes, and consequently decreases defaultable bond risk premia. The model thus distinguishes between systematic risk and the risk premium. When $S$ approaches zero the compensation per unit of risk rises but the risk exposure decreases. The latter effect dominates for very low levels of $S$ which reduces the bond risk premium as shown in Figure 3.

The impact of disaster-triggered default can also be understood from the strong positive relationship between the pricing kernel and default time. The pricing kernel is high and default is more 
likely upon the strike of consumption jumps. This relationship is one of two channels that generate high credit spreads. The relationship between consumption and the loss rate is another important channel in our model. We now discuss these in more detail.

\subsection{Countercyclical Loss Rates, Habit Persistence, and Bond Prices}

In this section we provide some intuition for how the jointly countercyclical dynamics in $L$ and $\gamma$ create large credit spreads. For this purpose we borrow from the insightful shorthand representation in CCDG for defaultable bonds which helps us explain the economic channels through which our model matches observed credit spreads. First, write the price of a zero coupon bond $P$ as

$$
P=E\left[\Lambda(A)\left(1-1_{D}(A, B) L(A, B)\right)\right]
$$

where $\Lambda$ represents the pricing kernel, $A$ denotes a vector of macro-economic variables including the surplus ratio, $S$, and where $B$ denotes a vector of firm specific (idiosyncratic) variables. The default indicator is denoted by $1_{D}(A, B)$. Note that we have suppressed the time subscripts everywhere. Expanding the expectation in (23) gives

$$
P=E[\Lambda(A)] E\left[1-1_{D}(A, B) L(A, B)\right]-\operatorname{Cov}\left(\Lambda(A), 1_{D}(A, B) L(A, B)\right),
$$

which we refer to as the expectation term and the covariance term, respectively.

Huang and Huang (2012) emphasize that both $E\left[1_{D}(A, B)\right]$ and $E[L(A, B)]$ should match their empirical levels when testing a model. With these constraints, and as emphasized by CCDG, it is clear that a large covariance between the pricing kernel and the expected loss in default is key to generating low defaultable bond prices and thus large credit spreads. Our model generates such a covariance via consumption-related default intensities and countercyclical loss rates.

Countercyclical loss rates also impact the expectation term in a desirable fashion. To see this we expand the expectation term in (24) to write

$$
\begin{aligned}
& E[\Lambda(A)] E\left[1-1_{D}(A, B) L(A, B)\right]=E[\Lambda(A)]\left[1-E\left[1_{D}(A, B) L(A, B)\right]\right] \\
& =E[\Lambda(A)]\left[1-E\left[1_{D}(A, B)\right] E[L(A, B)]-\operatorname{Cov}\left(1_{D}(A, B), L(A, B)\right)\right]
\end{aligned}
$$

which shows that a positive covariance between the default probability and the loss rate will drive down the expectation term and in turn decrease bond prices and increase credit spreads-even when constraining $E\left[1_{D}(A, B)\right]$ and $E[L(A, B)]$ to empirically relevant levels. A positive correlation between default rates and loss rates is a strong empirical regularity and it is captured in our model via the joint countercyclicality in defaults and loss rates arising from their covariance with the consumption process and thus with the habit process, $\gamma$.

In summary, allowing the loss rate to depend on macro conditions not only increases the covariance term as explained above but also decreases the expectation term. We thus conclude that 
accounting for a countercyclical loss rate that is correlated with the default intensity plays a double role in decreasing bond prices and generating nontrivial credit spreads.

\subsection{Credit Spreads and the Default Event Premium}

One may be concerned that the nontrivial credit spreads generated in our model are simply driven by an unrealistically large default event premium. In this section we show that this is not the case. The magnitude of macro disasters in our models, and thus the implied magnitude of pricing kernel jumps, $J_{\Lambda t}$, is calibrated to the international evidence on consumption disasters taking into account subsequent recoveries (Nakamura, Steinsson, Barro, and Ursua, 2011). Let $\lambda_{t}^{Q}$ denote the risk neutral expectation of the default intensity, $E_{t}^{Q}\left[d N_{t}\right]$, then the implied default intensity ratio between $P$ and $Q$ measures is

$$
\lambda_{t}^{Q} / \lambda=1+J_{\Lambda t}=e^{-J_{c}}\left(1-\alpha_{\gamma} \frac{\gamma_{t}-\beta}{\gamma_{t}} J_{c}\right)=e^{-J_{c}}\left(1-\alpha_{\gamma}\left(1-\beta S_{t}\right) J_{c}\right)
$$

which is linear in $S_{t}$ with slope $e^{-J_{c}} \alpha_{\gamma} \beta J_{c}=-150.66$ and intercept $e^{-J_{c}}\left(1-\alpha_{\gamma} J_{c}\right)=8.71$ in our calibration of the model. The default intensity ratio can be viewed as a measure of the default event premium.

Using an average surplus ratio of 0.03 the default intensity ratio is around 4 on average in the model. This number is in line with the empirical findings in Berndt, Douglas, Duffie, Ferguson, and Schranz (2008) who use physical default rates and CDS premia to estimate $\lambda_{t}^{Q} / \lambda$ ranging from 2.5 to 5.6 for investment grade firms. Our average default intensity ratio of 4 matches very well with these findings. The relatively modest average default intensity ratio also shows that our model's ability to generate realistic credit spreads is not due to the assumption of a large default (event) premium.

The default intensity ratio is strongly countercyclical in our model. A low surplus ratio of say $S_{t}=0.005$, implies a $\lambda_{t}^{Q} / \lambda$ of almost 8 which in turn denotes the high compensation per unit of bond price jump attributed to default. In our model, the default intensity under the $P$ measure is correlated with consumption jumps. Default risk is priced through the covariance between the default intensity and the consumption disaster intensity. The correlation of arrivals of default and arrivals of economic disaster can be computed from (12) to be $42.7 \%$ for Baa firms and $10 \%$ for Aaa firms.

We emphasize that a time-varying default rate under the $P$ measure is not required for a model to resolve the credit spread puzzle. What is necessary is that the occurrence of default covaries with consumption jumps and thus with the pricing kernel. In our habit-based model, the risk-neutral

expected default rate, $\lambda_{t}^{Q}$, is highly variable due the volatility of the surplus ratio, $S_{t}$, even though we have no variation in the physical default intensity, and even though our model-implied ratio on average matches the data.

Encouraged by our model's ability to create counter-cyclical credit spreads of sensible magni- 
tudes under realistic assumptions about physical default and the pricing kernel, we now proceed with a proper empirical assessment of our model.

\section{Model-Implied Versus Empirical Credit Spreads}

In this section we compare the model to empirically observed credit spreads. First, we compare the model's implications for average Corporate-Treasury spreads with those found in the data. Second, we analyze market and model-based Baa-Aaa spreads. Third, we analyze the term structure of BaaAaa spreads in the model and in the market data. Finally, we analyze the time-series properties of model-implied and market spreads.

\subsection{Average Corporate-Treasury Spreads}

On average, the model generates a 114 bps spread for Baa-Treasury and a 7 bps spread for AaaTreasury. These numbers are close to the average 5-year CDS spreads on Baa and Aaa rated firms which are 107 bps and 18.5 bps, respectively, when averaging Markit CDS spreads on the 125 constituent firms in the North American investment grade index during 2001-2010. The model spreads are lower than commonly observed corporate bond spreads. The latter discrepancy results from the fact that our model accounts solely for credit risk and abstracts from other factors that affect corporate bond prices such as taxes, conversion options, and illiquidity. Several papers have investigated the decomposition of spreads into various components and shown that the aforementioned factors are sizable. ${ }^{3}$ In light of these findings, we interpret our match for CDS averages and the underestimation for investment grade bonds as expected.

In a pure habit-formation setting, CCDG report a 4-year Aaa model-implied spread of 1 bps. In a long-run risk framework, Chen (2010) reports a model-implied spread of 45 bps for Aaa while the CDS spread for a similar rating is around $10 \mathrm{bps}$ and the corporate bond spread is around $53 \mathrm{bps}$. One reason for the differences in matching credit spreads between the long-run risk and existing habit literatures partly lies in the steeply upward-sloping yield curve in habit formation and the downward sloping yield curve in long-run risk models. Accounting for the macro disaster component in our external habit setup implies a moderately upward-sloping yield term-structure. This latter feature enables our model to match credit spreads when measured using CDS data.

In the remainder of this section we focus attention on Baa-Aaa spreads. Our reasoning, which follows the literature, is that if components of the credit spread due to non-default factors are of similar magnitude for Aaa and Baa bonds, then their relative spread should be mostly due to credit risk. We recognize that the call feature on Baa bonds may be more valuable than the call feature on Aaa bonds. Also, we know from Ericsson and Renault (2006) and He and Xiong (2012) that liquidity and credit are related. Nevertheless, the Baa-Aaa spread most likely captures credit risk

\footnotetext{
${ }^{3}$ See, for example, Elton, Gruber, Agrawal and Mann (2001), Driessen (2005), Longstaff, Mithal and Neis (2005), and Chen, Lesmond and Wei (2007).
} 
more accurately than do the corporate spreads versus Treasury.

\subsection{Average Baa-Aaa Spreads}

We now report on 5-year Baa-Aaa spreads under the four different versions of the model discussed in Section 3.3. In Table 2.A we use the stationary distribution of the state variables in the model to compute the model-implied Baa-Aaa spreads.

Table 2.A: Baa-Aaa Spreads (bps). Stationary Distribution.

\begin{tabular}{llll}
\hline Model Version & Constraints & Spread Mean & Spread Vol. \\
\hline Constant Loss and Habit & $k_{\gamma}=\alpha_{\gamma}=k_{L}=\alpha_{L}=0$ & 23.0 & 0 \\
Constant Habit & $k_{\gamma}=\alpha_{\gamma}=0$ & 36.2 & 1.96 \\
Constant Loss Rate & $k_{L}=\alpha_{L}=0$ & 49.5 & 9.74 \\
Full Model & None & 107 & 40.7 \\
\hline & Empirical Values from Markit & 88.5 & 37.4 \\
\hline \hline
\end{tabular}

Table 2.A shows that the Full Model, which incorporates a countercyclical loss rate, matches the average and the standard deviation of credit spreads quite well. This result again emphasizes the importance of the consumption disaster in addition to both countercyclical habit and loss rate. When viewed individually, time variation in habit (Constant Loss Rate) is better able to generate credit spread volatilities than is time variation in the loss rate (Constant Habit). Notice again that-due to the nonlinearity in the model-the effect of habit persistence together with the loss rate is larger than the sum of their individual effects.

So far we have documented results for 5-year Baa-Treasury and Aaa-Treasury spreads, and also for 5-year Baa-Aaa spreads. We now turn our attention to the performance of the model in matching the observed spread term structure.

\subsection{The Term Structure of Baa-Aaa Spreads}

Figure 4 shows the average term structure of Baa-Aaa spreads from 2001 to 2010 (solid line) along with the model-implied credit spreads for various special cases of the model. We know from Table 2.A above that only the full version of the model is able to come close to matching the 5-year credit spreads. Figure 4 confirms this. Furthermore, Figure 4 shows that the full model is able to roughly match the average slope and shape of the credit spread term structure.

The model with constant loss rate implies a declining term structure of credit spreads for maturities beyond 4 years. The model with constant loss rate and habit both exhibit spread levels that are too low on average and a spread term structure that is too flat on average. The model with constant habit but dynamic loss rate appears to match the slope of the credit term structure quite well but of course misses the credit spread levels completely.

The short-term credit spread is strictly positive and sizable in our full model for the following reasons. First, relating the default of investment grade bonds to macro disasters makes default a 
nonzero probability event in the limit in our setup. This is a desirable property of the incomplete information framework, including that in Chen and Kou (2009) and Duffie and Lando (2001). Second, default for investment grade bonds coincides with states of high marginal utility because risk aversion also peaks around default events. These effects both help generate an empirically relevant spread for short maturities. The crucial insight is that habit models augmented with macro disasters-along with the recognition that investment grade bonds tend to default during bad macro conditions-are able to match observed short term spreads quite well.

Traditional structural models usually generate extremely steep credit spread term structures. As mentioned in Duffie and Lando (2001), this is due to the fact that, for models producing a zero short-term spread in the limit, a steep slope is required to match observed spreads for medium term bonds. As noted above, our full model matches the short term spread quite well. Consequently, as illustrated in Figure 4, the model does not induce excessive steepness in order to achieve a reasonable spread for medium term bonds.

An additional advantage of our approach is that matching the medium-term spreads does not come at the expense of generating an unrealistic downward sloping term structure for longer maturities so long as we allow for counter-cyclical loss rates as Figure 4 shows. For long maturities the model-implied term-structure of credit spreads tends to flatten in the full model which is also observed empirically. This is a desirable property of modelling default in an incomplete information setup as well as having stochastic recovery compounding the effect of a loss when it occurs in a bad state of the economy. In contrast, modelling default as the first hitting time of an asset boundary often leads to a convergence to zero of spreads for long maturities, which is due to the stationarity of default boundaries and to the risk-neutral firm cash-flows growing at a positive expected rate. Such models imply a strong downward sloping term structures beyond the medium term.

Our model has the ability to generate both upward and humped-shaped term structures depending on the parameter values. Humped-shaped credit term structures have been documented empirically in Jarrow, Lando and Turnbull (1997) who find that term structures are upward sloping for credits rated A or better but that they can be slightly humped for Baa rated credits.

We highlight again that our full model is able to deliver an average term structure of credit spreads that matches key stylized facts even though the model is calibrated to a single set of parameters that is fixed across all maturities. In particular, the default intensity $\lambda_{j}$ is calibrated

in Table 1.C to physical default probabilities from Moody's for each of the two credit classes we consider.

\subsection{Time Series Dynamics in the Baa-Aaa Spread}

Our model's ability to match the second moments of Baa-Aaa spreads as in Table 2.A and the term structure of credit spreads in Figure 4 bodes well for our model's ability to capture the time series dynamics in observed spreads. To investigate this dimension of the data, following CCDG, we obtain the time series of past innovations in log consumption growth from the St Louis Fed 
(post-1952) and Robert Shiller's website (pre-1952). Following Campbell and Cochrane (1999) and Menzly, Santos and Veronesi (2004), we use this innovation to construct the time series of the surplus ratio and loss rates using (1)-(2) and (13), respectively. Given the time series of $\gamma$ and $L$, we back out model-implied credit spreads over time and compare them to historical data.

Using credit spread data from the St. Louis Fed for the period 1919-2010, Figure 5 plots in each panel the observed historical credit spread (solid line) along with one of the four special cases of the model (dashed lines). The St. Louis Fed relies on data from Moody's Investor Services which includes bonds with remaining maturity higher than 20 years and as close as possible to 30 years. The top-left panel shows that when both loss rate and habit are constant then model spreads are constant and low. The top-right panel shows that with constant habit, a dynamic loss rate is only able to generate minor variations in model spreads and the levels are still much too low. The bottom-left panel of Figure 5 shows that with a constant loss rate, the dynamic habit is able to generate some dynamics in model spreads but arguably not enough and the level still appears to be too low. Finally, the full model in the bottom-right panel of Figure 5 shows that when the loss rate and habit are both dynamic then the model is able to capture the level and wide fluctuations in observed spreads.

Table 2.B below reports the various sample moments for the model and market spreads.

Table 2.B: Baa-Aaa Spreads (bps). Time Series Moments.

\begin{tabular}{lllllll}
\hline Model Version & Constraints & Mean & Vol & Min & Max & RMSE \\
\hline Constant Loss and Habit & $k_{\gamma}=\alpha_{\gamma}=k_{L}=\alpha_{L}=0$ & 25.5 & 0 & 25.5 & 25.5 & 5.033 \\
Constant Habit & $k_{\gamma}=\alpha_{\gamma}=0$ & 39.5 & 4.3 & 29.5 & 56.0 & 2.674 \\
Constant Loss Rate & $k_{L}=\alpha_{L}=0$ & 75.9 & 33.9 & 36.3 & 180.5 & 1.254 \\
Full Model & None & 129.0 & 80.1 & 42.6 & 431.6 & 0.686 \\
\hline & Empirical Values (St. Louis Fed) & 126.1 & 79.8 & 34.0 & 510.0 & \\
\hline \hline
\end{tabular}

Table 2.B confirms the visual impression from Figure 5 that only the full model is able to match the level of observed historical credit spreads. Table 2.B also shows that the full model has the lowest root mean squared error (RMSE) which we compute on the relative credit spread errors as follows

$$
R M S E=\sqrt{\frac{1}{T} \sum_{t=1}^{T}\left(\left(C S_{t}^{M k t}-C S_{t}^{M o d}\right) / C S_{t}^{M k t}\right)^{2}},
$$

where $C S_{t}^{M k t}$ and $C S_{t}^{M o d}$ denote market and model credit spreads, respectively. The full model is also the only model that is close to matching the maximum spread of 510 bps observed in the data.

We again stress the fact that our model parameters have not been fitted to bond prices. The exercise in Table 2.B and Figure 5 thus demonstrates the model's ability to fit the dynamics in credit spreads out-of-sample and it also highlights that both habit persistence and counter-cyclical loss rates are important for capturing observed spreads. 


\section{$5 \quad$ Exploring the Model Further}

In this section we explore various additional aspects of our model. In the empirics so far, we have set the idiosyncratic default intensity to zero. In the first part of this section we therefore explore the implications for credit spreads when default can be partly idiosyncratic in nature. We then study the impact on credit spreads of varying some of the key parameters in our model. Finally, we discuss the implications of our model for the joint pricing of defaultable bonds and index options.

\subsection{The Impact of Idiosyncratic Default on Credit Spreads}

When implementing the model in the previous sections, we set idiosyncratic default to zero, which is motivated by Bakshi, Madan, and Zhang (2006) who document empirically that high grade bond defaults are mainly explained by systematic factors. As a robustness check, we want to provide insights concerning the sensitivity of our model's performance to idiosyncratic shocks. Unfortunately, there are not many observed defaults for investment grade bonds, and even fewer default cases that do not coincide with recessionary times for high-quality credit. We thus resort to a numerical experiment designed to answer the following question: How large a fraction of idiosyncratic default is needed to match observed credit spreads?

Our benchmark calibration assumes that the idiosyncratic default is zero. Given that our model generates Aaa-Treasury spreads that match the average CDS spread quite well, the implied idiosyncratic fraction for this credit class is indeed close to zero.

The top panel of Figure 6 plots the model-implied Baa-Treasury spread against the ratio of idiosyncratic default to total default, $\lambda_{j}^{i d i o} /\left(\lambda_{j}^{s y s}+\lambda_{j}^{i d i o}\right)$ in percent. From this figure we see that the empirical average Baa-Treasury spread of 107 bps is matched in the model when idiosyncratic default constitutes about $5 \%$ of total default for Baa rated bonds.

We have performed the above exercise for eight different rating classes ranging form Aaa to Caa for which Moody's objective default probability and average CDS spreads are available. We find that the required idiosyncratic fraction increases as credit quality decreases: The required idiosyncratic component is indeed minor for all investment grade bonds, and it gets higher for speculative grade and for very low credit ratings it is the most important driver of default. These results are not reported but they are of course available upon request.

An important insight from this exercise is that our model produces a well-documented empirical fact: The implied ratio of default intensity between $P$ and $Q$ measure decreases with credit quality (e.g., Berndt et al., 2008, and Coval, Jurek and Stafford, 2009). To see this, note that we can use the decomposition $\lambda_{j}=\lambda_{j}^{s y s}+\lambda_{j}^{\text {idio }}$ and equation (25) to write the risk-neutral default intensity ratio as

$$
\lambda_{j t}^{Q} / \lambda_{j}=\frac{\left(1+J_{\Lambda t}\right)\left(\lambda_{j}-\lambda_{j}^{i d i o}\right)+\lambda_{j}^{i d i o}}{\lambda_{j}}=1+J_{\Lambda t}\left(1-\lambda_{j}^{i d i o} / \lambda_{j}\right),
$$

which is clearly decreasing in $\lambda_{j}^{i d i o}$. In particular, $\lambda_{j t}^{Q} / \lambda_{j}=1+J_{\Lambda t}>1$ when $\lambda_{j}^{i d i o}=0$ and 
$\lambda_{j t}^{Q} / \lambda_{j}=1$ when $\lambda_{j}^{i d i o}=\lambda_{j}$.

In the bottom panel of Figure 6 we plot the default intensity ratio against the fraction of idiosyncratic default, which shows a downward sloping pattern as the idiosyncratic component becomes larger which in turn occurs for the lower credit classes, as argued above.

The analysis in this section suggests that our model can match observed credit spreads across credit ratings. We focus on investment grade firms simply because they have proven to be the most challenging to match in the literature.

\subsection{Model Robustness Analysis}

In this section we first investigate the sensitivity of model-implied spreads to some key parameters. We focus on $\alpha_{\gamma}$ and $\alpha_{L}$ which control the response of habit and loss rates to consumption innovations. We also consider $\left|J_{c}\right|$ which captures the absolute log consumption jump size. These parameters play important roles in the three key features of our model, namely, consumption disasters, time-varying risk aversion, and counter-cyclical loss rates. We vary each of the three parameters by up to $30 \%$ in both directions from their calibrated values in Table 1, and plot the implied average and standard deviation of model-implied 5-year Baa-Aaa spreads in Figure 7.

The direction of changes in spreads and volatilities are as expected. By comparison, $\left|J_{c}\right|$ has the largest impact. This result re-emphasizes the importance of accounting for consumption disasters, when explaining investment grade credit spreads. The importance of the jump component can also be inferred from the long-run risk literature. Chen (2010) generates a realistically high average credit spread for 10-year Baa bonds. Like our model, Chen emphasizes the effect of a large jump risk premium on the pricing of defaultable bonds. In particular, by shutting down jumps, Chen reports a roughly $50 \%$ reduction in the generated spread, which is very much in line with our numerical results.

While our model features a constant $J_{c}$, we find that the model predictions are insensitive to the introduction of randomness in $J_{c}$. The reason can be found in the structure of the Menzly, Santos and Veronesi (2004) habit formation used in this paper which implies that the volatility of $J_{c}$ has little impact on the jump risk premium. Since the jump risk premium is closely related to the default risk premium in our setup, the implied credit spreads are also relatively insensitive to the volatility of $J_{c}$.

We next investigate if our results are driven by the specific Menzly, Santos and Veronesi (2004) MSV-habit that we assume. We consider the SV-habit specification by Santos and Veronesi (2010) which is close to the original Campbell and Cochrane (1999) CC-habit but with an extra parameter controlling utility curvature. At first sight, one may argue that having an extra parameter will surely enhance the model's ability to capture prices in-sample. However, this is not the case in our exercise since our model is not fitted to bond prices. The matching of credit spreads is thus a true outof-sample exercise which may actually be more difficult with a richer parameterization. Choosing SV-habit instead of MSV-habit and showing that our results still hold demonstrates that our model 
performance is not driven by the MSV-habit that we focus on in this paper. We solve for all asset valuations in this new framework. We consider various utility curvature parameter values under SV habit. To save space we do not report the results, which are available upon requests. We find that the SV-habit augmented with a consumption disaster component is also able to match empirical credit spreads in terms of their levels, term-structure and time series dynamics. Consequently, we conclude that our results are not sensitive to changes in the habit specification.

\subsection{Empirical Option Skewness Dynamics}

We now provide an alternative empirical check on our model by assessing its ability to capture observed time series variation in option skewness. Du (2011) fits the consumption-based asset

pricing model in this paper to index option prices and finds that the model matches the key unconditional moments including the level of option skewness. We extend his analysis and focus on the joint pricing of options and defaultable bonds. Du (2011) shows that when assuming aggregate dividends equal consumption the aggregate equity price can be derived as

$$
P_{t}^{E}=\left(\frac{1}{\rho+k_{\gamma}}+\frac{k_{\gamma} \bar{\gamma}}{\rho\left(\rho+k_{\gamma}\right)} \frac{1}{\gamma_{t}}\right) C_{t}
$$

Using our parameter calibration, the equity risk premium is $5.9 \%$ per year and the annual stock market volatility is $17.6 \%$ on average. Both values match empirical observations very well. Option prices can be computed in the model via Monte Carlo simulation of consumption and stock prices.

We obtain daily S\&P 500 index option data from CBOE and Ivy DB which combine to cover a period of nearly 25 years from April 4, 1988 to October 29, 2010. We then compute the modelimplied time series of option skewness for one-month contracts, which are compared to their empirical counterparts during the sample period.

We consider three different measures of option skewness: Besides the usual measure computed as the Black-Scholes implied volatility $(I V)$ difference between 10\% OTM put options and ATM options, we also consider option skewness for $8 \%$ OTM puts and $6 \%$ OTM puts, that is

$$
\begin{aligned}
O S_{1, t} & \equiv I V_{t}(M=.90)-I V_{t}(M=1) \\
O S_{2, t} & \equiv I V_{t}(M=.92)-I V_{t}(M=1) \\
O S_{3, t} & \equiv I V_{t}(M=.94)-I V_{t}(M=1)
\end{aligned}
$$

where $M$ is the option moneyness defined as strike price over stock price.

Table 3 below reports the results based on the following time series regression

$$
O S_{i, t}^{\text {Market }}=\beta_{1}+\beta_{2} O S_{i, t}^{\text {Model }}+\epsilon_{i, t}, \text { for } i=1,2,3,
$$

where superscript Market and Model denote option skewness from observed option prices and model-implied option prices, respectively. 


\begin{tabular}{|c|c|c|c|}
\hline Skewness Measure & $\beta_{2}$ & $\mathrm{NW} t\left(\beta_{2}\right)$ & $R^{2}$ \\
\hline$O S_{1}: 10 \%$ OTM IV & 0.583 & 9.469 & 0.457 \\
\hline $8 \%$ OTM IV & 0.646 & 9.201 & 0.351 \\
\hline $6 \%$ OTM IV & 0.750 & 6.305 & 0.241 \\
\hline
\end{tabular}

Table 3 shows that the $\beta_{2}$ coefficients are economically significant taking on values between 0.583 and 0.750 . These values are highly statistically significant when computing t-stats using Newey-West standard errors adjusted for heteroskedasticity and autocorrelation. Furthermore, the $R^{2}$ measures are also sizable. We thus conclude that our model does a fairly good job in capturing the time series variations of the observed option skewness.

\subsection{Credit Spreads, Option Skewness and Disaster Magnitude}

Our model's resolution of credit spread puzzles hinges on the consumption disaster component, which drives jumps in the pricing kernel, and on relating defaults to consumption disasters. It is well known from the option literature that a jump component is also crucial for index option pricing. It is therefore interesting to study our model's ability to jointly fit bond spreads and option prices.

To provide a graphical illustration, we vary the absolute consumption jump size $\left|J_{C}\right|$, and plot in the top two panels of Figure 8 the implied average Baa-Aaa credit spread and the average option skewness using the $O S_{1}$ definition in (27) above. Both the Baa-Aaa spread and option skewness rise monotonically with $\left|J_{C}\right|$ : For defaultable bonds, a larger $\left|J_{C}\right|$ means a higher price of default risk which drives up the default-risk premium and hence the credit spread. For equity index options, a larger $\left|J_{C}\right|$ implies a higher jump risk premium for aggregate equity, which generates a larger option skewness. The empirical relevant option skewness is around 9\% (Dumas, Fleming and Whaley, 1998, and Du, 2011) and it is reached only when consumption jumps are close to $15 \%$ in magnitude.

To give a more direct comparison, we plot in the bottom panel of Figure 8 the average option skewness against the average credit spread as we again vary $\left|J_{C}\right|$. In the absence of jumps $\left(\left|J_{C}\right|=0\right)$, habit formation can still produce option skewness but it is very small. Simultaneously, the implied credit spread is less than one-fifth of the observed level. Both rise in lockstep as we increase the consumption jump severity until they reach their respective empirical level at our base case calibration of consumption jump, when $\left|J_{C}\right|=0.15$. Thus, we conclude that a disaster component is crucial for linking credit and option markets.

\subsection{Credit Spreads, Option Skewness and the State of the Economy}

To understand how the spread/skewness relationship varies by the state of the economy, we carry out comparative statics with respect to the state of the economy as proxied by different levels of 
the surplus ratio. Specifically, we plot in the top two panels of Figure 9 the state dependence of

the 5-year Baa-Aaa spreads and option skewness. As discussed previously, the credit spread in the top panel is clearly countercyclical. Indeed, recently economists have argued that average credit spreads can be used to forecast economic growth (Gilchrist and Zakrajšek, 2012).

Option skewness in the middle panel, however, is highly nonlinear in the surplus ratio; it is decreasing at very low levels of $S$, and then becomes increasing for higher $S$ levels. This is because an increase in the surplus ratio has two offsetting effects on option skewness: First, it decreases the severity of consumption jumps under the pricing measure which gives the agent less incentives to hedge, hence the lower option premium for a given diffusive stock volatility. Second, it decreases the diffusive stock volatility which increases the relative importance of jumps in the total stock price variation. As a result, the agent has stronger incentives to buy insurance via OTM puts which drives up option skewness. The first effect dominates when $S$ is small, hence the higher premium as the economy gets worse. However, for relatively large $S$, the second effect dominates leading to increasing option skewness in $S$ when $S$ is high.

It is interesting to contrast the bottom panel of Figure 8 with the bottom panel of Figure 9: Figure 8 shows that there is an almost linear relationship between credit spreads and option skewness when we increase the consumption jump size from 0 to the benchmark calibration of $15 \%$ per year. But Figure 9 shows that there is a non-linear relationship between credit spreads and option skewness when increasing the surplus ratio, that is, when going from a very bad to a very good state of the economy.

The above analysis suggests that, while credit spreads may proxy for macroeconomic conditions, this is not the case for option skewness. Our model shows that option skewness cannot be viewed as a reliable proxy for credit spreads as both skewness and spreads are endogenously determined by their respective exposure to consumption disasters. In addition, our model clearly suggests a nonlinear relation between the two variables. Running regressions of credit spreads on option skewness, as is sometimes done in the literature (e.g., Cremers, Driessen, Maenhout, and Weinbaum, 2008), is likely to lead to inconclusive results that depend on the state of the economy in the sample at hand.

\section{Conclusion}

We have shown that it is possible to explain key stylized facts in credit markets in a model of rare macro disasters, when using a single set parameters calibrated only to the real economy, and when restricting disasters to be of reasonable size. Our model is able to match the relative high average level and volatility of investment grade credit spreads for short and medium-term bonds, the upward-sloping average term structure of investment grade credit spreads, the strongly persistent and highly non-linear time series dynamics in credit spreads, and the time-varying and non-linear relationship between credit spreads and option skewness. 
The stylized facts in credit markets are captured in our rare-disaster model that incorporates habit persistence, counter-cyclical default and loss rates. Importantly, we provide strong evidence that all of these features are required in order to match the stylized facts.

Several interesting extensions are left for future work. We have emphasized the value of matching key moments in credit spreads and options using a single set of parameters calibrated to the real economy. However, going forward, it would be of interest to estimate the full model on observed credit spreads and option prices in order to provide a more detailed empirical assessment of the model. Our closed-form solutions for defaultable bond prices will be very valuable in this regard.

While we have allowed for dynamic habit and recovery in our model, we have assumed that physical default intensities are constant over time. It would be interesting to relax this assumption. However, the scarcity of investment grade defaults in the data will render identification and estimation of time-varying default a challenge. The derivation of closed-form bond prices will also be difficult in an environment where loss rates, habit and default rates are all stochastic.

\section{Appendix: Proof of Propositions}

In Lemma 1 below we derive the price of a defaultable bond price under the simplifying assumption that the recovery payment is paid on the terminal payment (TP) date of the bond. We then prove Proposition 1 as a special case of Lemma 1. More importantly, we show how Proposition 2 can be proved as a generalization of the intermediate TP case in Lemma 1. Finally, Proposition 3 is proved using Ito's lemma for jump-diffusions. The bond pricing formulas we present below are quite involved and we have therefore verified their accuracy via Monte Carlo simulation.

Lemma 1 Under the TP assumption, the defaultable bond price takes the form

$$
P_{t, \tau}^{T P}=\alpha_{1}^{T P}(\tau)+\alpha_{2}^{T P}(\tau) \frac{1}{\gamma_{t}}+\alpha_{3}^{T P}(\tau) L_{t}+\alpha_{4}^{T P}(\tau) \frac{L_{t}}{\gamma_{t}}
$$

where the expressions for $\alpha_{i}^{T P}$ are given in the proof below.

Before proceeding with the proofs a couple of definitions are needed. For a given rating class, $j$, the potential default of the bonds can be written in transition matrix form as

$$
\Gamma_{j}^{s y s} \equiv\left[\begin{array}{cc}
-\lambda_{j}^{s y s} & \lambda_{j}^{s y s} \\
0 & 0
\end{array}\right] \text { and } \Gamma_{j}^{i d i o} \equiv\left[\begin{array}{cc}
-\lambda_{j}^{i d i o} & \lambda_{j}^{i d i o} \\
0 & 0
\end{array}\right]
$$

where the first regime is no-default and the second regime is default, which is absorbing. We suppress the rating subscript, $j$, below.

Note that the proofs in this Appendix are based on the following generalized version of the loss rate process:

$$
d L_{t}=k_{L}\left(\bar{L}-L_{t}\right) d t-\alpha_{L} \sigma d \tilde{B}_{t}-\alpha_{L} b\left(d N_{t}-\lambda_{c} d t\right)
$$


where $\tilde{B}_{t}$ is an additional standard Brownian which has correlation $\tilde{\rho}$ with the consumption Brownian, $B_{t}$. This specification allows for $L_{t}$ to be driven by an idiosyncratic shock in addition to the systematic consumption shock.

\section{A.1 Proof of Lemma 1: Recovery on the Terminal Payment Date}

Let $P_{t, \tau}^{T P}\left(s_{t}\right)$ be the price of a zero-coupon defaultable bond with $\tau$ period to expiration, where $P_{t, \tau}^{T P}(1)$ and $P_{t, \tau}^{T P}(2)$ denote prices conditional on being in the first (no-default) and the second (default) regime, respectively. When normalizing the face value of the bond to 1 , and using the definition of TP, we have

$$
P_{t, \tau}^{T P}\left(s_{t}\right)=\left\{\begin{array}{ll}
E\left[\frac{\Lambda_{T}}{\Lambda_{t}} \mid s_{t}\right], & \text { if } s_{T}=1 \\
E\left[\frac{\Lambda_{T}}{\Lambda_{t}}\left(1-L_{T}\right) \mid s_{t}\right], & \text { if } s_{T}=2
\end{array},\right.
$$

where $\Lambda_{T}$ and $L_{T}$ denote the pricing kernel and the loss rate at the terminal date $T$ and where we have used the fact that the default regime is absorbing. Using the expression for the pricing kernel in $(7)$, we can write

$$
P_{t, \tau}^{T P}\left(s_{t}\right)=\frac{C_{t}}{\gamma_{t}}\left(E\left[e^{-\rho \tau} \theta^{c}\left(s_{T}\right) C_{T}^{-1} \gamma_{T} \mid s_{t}\right]+E\left[e^{-\rho \tau} \theta^{s}\left(s_{T}\right) C_{T}^{-1} \gamma_{T} L_{T} \mid s_{t}\right]\right)
$$

where we have stacked the regimes using

$$
\theta^{c} \equiv\left[\begin{array}{l}
\theta^{c}(1) \\
\theta^{c}(2)
\end{array}\right]=\left[\begin{array}{l}
1 \\
1
\end{array}\right], \text { and } \theta^{s} \equiv\left[\begin{array}{l}
\theta^{s}(1) \\
\theta^{s}(2)
\end{array}\right]=\left[\begin{array}{c}
0 \\
-1
\end{array}\right] \text {. }
$$

We can rewrite (A.5) as

$$
P_{t, \tau}^{T P}\left(s_{t}\right)=\frac{C_{t}}{\gamma_{t}}\left[\psi^{c}\left(x_{t} ; s_{t}\right)+\psi^{s}\left(x_{t} ; s_{t}\right)\right]
$$

where we have defined,

$$
\begin{aligned}
\psi^{c}\left(x_{t}, \tau ; s_{t}\right) & \equiv E\left[e^{-\rho \tau} x_{1 T} x_{2 T} \theta^{c}\left(s_{T}\right) d u \mid s_{t}\right] \\
\psi^{s}\left(x_{t}, \tau ; s_{t}\right) & \equiv E\left[e^{-\rho \tau} x_{1 T} x_{2 T} x_{3 T} \theta^{s}\left(s_{T}\right) d u \mid s_{t}\right] .
\end{aligned}
$$

For ease of exposition we now define a vector of state variables, $x_{t}$, where

$$
x_{1 t} \equiv C_{t}^{-1} ; x_{2 t} \equiv \gamma_{t} ; x_{3 t} \equiv L_{t} .
$$

Using Ito's lemma for jump-diffusions, we can write

$$
d x_{j t}=\left(a_{j}+b_{j} x_{j t}\right) d t+\left(c_{j}+d_{j} x_{j t}\right) d B_{t}+\left(e_{j}+f_{j} x_{j t}\right) d N_{t},
$$


for $j \in\{1,2,3\}$, where

$$
\begin{array}{llllll}
a_{1}=0 & b_{1}=-\mu+\sigma^{2} & c_{1}=0 & d_{1}=-\sigma & e_{1}=0 & f_{1}=e^{-\gamma J_{c}}-1 \\
a_{2}=k_{\gamma} \bar{\gamma}-\alpha_{\gamma} \beta J_{c} \lambda_{c} & b_{2}=-k_{\gamma}+\alpha_{\gamma} J_{c} \lambda_{c} & c_{2}=\alpha_{\gamma} \beta \sigma & d_{2}=-\alpha_{\gamma} \sigma & e_{2}=\alpha_{\gamma} \beta J_{c} & f_{2}=-\alpha_{\gamma} J_{c} \\
a_{3}=k_{L} \bar{L}+\alpha_{L} J_{c} \lambda_{c} & b_{3}=-k_{L} & c_{3}=-\alpha_{L} \sigma & d_{3}=-\sigma & e_{3}=-\alpha_{L} J_{c} & f_{3}=0
\end{array} .
$$

We proceed with the derivation of $\psi^{s}\left(x_{t}, \tau ; i\right)$. By applying the multi-regime version of FeynmanKac theorem (e.g., Mao and Yuan, 2006), $\psi^{s}\left(x_{t}, \tau ; i\right)$ defined in (A.9) is the solution to the following partial differential equation (PDE):

$$
\rho \psi^{s}\left(x_{t}, \tau ; i\right)=\Im \psi^{s}\left(x_{t}, \tau ; i\right) \text {, subject to } \psi^{s}\left(x_{T}, 0 ; i\right)=\theta^{s}(i) x_{1 T} x_{2 T} x_{3 T} \text {. }
$$

Stacking the regimes yields

$$
\rho \psi^{s}\left(x_{t}, \tau\right)=\Im \psi^{s}\left(x_{t}, \tau\right) \text { subject to } \psi^{s}\left(x_{T}, 0\right)=\theta^{s} x_{1 T} x_{2 T} x_{3 T},
$$

where

$$
\begin{aligned}
\Im \psi^{s}\left(x_{t}, \tau\right) \equiv & -\frac{\partial \psi^{s}(.)}{\partial \tau}+\sum_{j=1}^{3} \frac{\partial \psi^{s}(.)}{\partial x_{j t}}\left(a_{j}+b_{j} x_{j t}\right)+\frac{1}{2} \sum_{j, j^{\prime}} \rho_{j j^{\prime}} \frac{\partial^{2} \psi^{s}(.)}{\partial x_{j t} \partial x_{j^{\prime} t}}\left(c_{j}+d_{j} x_{j t}\right)\left(c_{j^{\prime}}+d_{j^{\prime}} x_{j^{\prime} t}\right) \\
& +\lambda_{c}\left[\psi^{s}\left(x_{t}+\Delta\left(x_{t}\right), \tau\right)-\psi^{s}\left(x_{t}, \tau\right)\right]+\Gamma^{s y s} \psi^{s}\left(x_{t}+\Delta\left(x_{t}\right), \tau\right)+\Gamma^{i d i o} \psi^{s}\left(x_{t}\right), \quad(\mathrm{A} .13)
\end{aligned}
$$

and where

$$
\begin{gathered}
\rho_{13}=\rho_{23}=\tilde{\rho} \text { while } \rho_{j j^{\prime}}=1 \text { for all other }\left(j, j^{\prime}\right) \text { pairs, and } \\
\Delta\left(x_{t}\right) \equiv\left[e_{1}+f_{1} x_{1 t}, e_{2}+f_{2} x_{2 t}, e_{3}+f_{3} x_{3 t}\right]^{\prime}
\end{gathered}
$$

We have used the fact that the systematic defaults are triggered by $x$-jumps which themselves are induced by consumption jumps in our model.

We conjecture-and later verify-that (A.12)-(A.13) has the following solution:

$$
\psi^{s}\left(x_{t}, \tau\right)=\eta_{111}^{s}(\tau) x_{1 t} x_{2 t} x_{3 t}+\eta_{101}^{s}(\tau) x_{1 t} x_{3 t}+\eta_{110}^{s}(\tau) x_{1 t} x_{2 t}+\eta_{100}^{s}(\tau)
$$

It can be verified that all terms in $\Im \psi^{s}\left(x_{t}, \tau\right)$ are proportional to $x_{1 t} x_{2 t} x_{3 t}$, or $x_{1 t} x_{3 t}$, or $x_{1 t} x_{2 t}$, or 1 , from which we can identify $\eta(\tau)$. More specifically, collecting the terms in (A.12)-(A.13) that are proportional to $x_{1 t} x_{2 t} x_{3 t}, x_{1 t} x_{3 t}, x_{1 t} x_{2 t}$, and 1 , yields the following ordinary differential equations (ODEs):

$$
\frac{d \eta_{h}^{s}(\tau)}{d \tau}=-A_{h}^{s} \eta_{h}^{s}(\tau)+B_{h}^{s}(\tau) \text { subject to } \eta_{h}^{s}(0)=\theta_{h}^{s}
$$

where $h \in\{111,101,110,100\}$;

$$
\theta_{111}^{s}=\theta^{s} \text { and } \theta_{h}=0_{2 \times 1} \text { for } h \neq 111
$$


$A_{h}^{s}$ and $B_{h}^{s}(\tau)$ are given by:

$$
\begin{aligned}
A_{111}^{s}= & \beta-b_{1}-b_{2}-b_{3}-\rho_{12} d_{1} d_{2}-\rho_{13} d_{1} d_{3}-\rho_{23} d_{2} d_{3} \\
& -\lambda_{c}\left[\left(1+f_{1}\right)\left(1+f_{2}\right)\left(1+f_{3}\right)-1\right]-\left(1+f_{1}\right)\left(1+f_{2}\right)\left(1+f_{3}\right) \Gamma^{s y s}-\Gamma^{\text {idio }}, \\
A_{101}^{s}= & \rho-b_{1}-b_{3}-\rho_{13} d_{1} d_{3}-\lambda_{c}\left[\left(1+f_{1}\right)\left(1+f_{3}\right)-1\right]-\left(1+f_{1}\right)\left(1+f_{3}\right) \Gamma^{s y s}-\Gamma^{i d i o}, \\
A_{110}^{s}= & \rho-b_{1}-b_{2}-\rho_{12} d_{1} d_{2}-\lambda_{c}\left[\left(1+f_{1}\right)\left(1+f_{2}\right)-1\right]-\left(1+f_{1}\right)\left(1+f_{2}\right) \Gamma^{s y s}-\Gamma^{i d i o}, \\
A_{100}^{s}= & \rho-b_{1}-\lambda_{c} f_{1}-\left(1+f_{1}\right) \Gamma^{\text {sys }}-\Gamma^{i d i o},
\end{aligned}
$$

and

$$
\begin{aligned}
B_{111}^{s}(\tau)= & 0_{2 \times 1} \\
B_{101}^{s}(\tau)= & {\left[a_{2}+\rho_{12} c_{2} d_{1}+\rho_{23} c_{2} d_{3}+\lambda_{c}\left(1+f_{1}\right)\left(1+f_{3}\right) e_{2}\right] \eta_{111}^{s}(\tau)+\left(1+f_{1}\right)\left(1+f_{3}\right) e_{2} \Gamma^{s y s} \eta_{111}^{s}(\tau), } \\
B_{110}^{s}(\tau)= & {\left[a_{3}+\rho_{13} c_{3} d_{1}+\rho_{23} c_{3} d_{2}+\lambda_{c}\left(1+f_{1}\right)\left(1+f_{2}\right) e_{3}\right] \eta_{111}^{s}(\tau)+\left(1+f_{1}\right)\left(1+f_{2}\right) e_{3} \Gamma^{s y s} \eta_{111}^{s}(\tau), } \\
B_{100}^{s}(\tau)= & {\left[a_{2}+\rho_{12} c_{2} d_{1}+\lambda_{c}\left(1+f_{1}\right) e_{2}\right] \eta_{111}^{s}(\tau)+\left[a_{3}+\rho_{13} c_{3} d_{1}+\lambda_{c}\left(1+f_{1}\right) e_{3}\right] \eta_{111}^{s}(\tau) } \\
& +\left[\rho_{23} c_{2} c_{3}+\lambda_{c}\left(1+f_{1}\right) e_{2} e_{3}\right] \eta_{111}^{s}(\tau)+\left(1+f_{1}\right) \Gamma^{s y s}\left[e_{2} \eta_{111}^{s}(\tau)+e_{3} \eta_{111}^{s}(\tau)+e_{2} e_{3} \eta_{111}^{s}(\tau)\right] .
\end{aligned}
$$

Solving (A.17) yields:

$$
\eta_{h}^{s}(\tau)=\int_{0}^{\tau} e^{-(\tau-u) A_{h}^{s}} B_{h}^{s}(u) d u+e^{-\tau A_{h}^{s}} \theta_{h}^{s}
$$

for $h \in\{111,101,110,100\}$ where $e^{A}$ denotes the matrix exponential. Substituting (A.11), (A.14), and (A.18) into (A.19)-(A.20) yields

$$
\begin{aligned}
& A_{111}^{s}=a_{111}^{s} I_{2 \times 2}-e^{-J_{c}}\left(1-\alpha_{\gamma} J_{c}\right) \Gamma^{s y s}-\Gamma^{i d i o}, \\
& A_{101}^{s}=a_{101}^{s} I_{2 \times 2}-e^{-J_{c}} \Gamma^{s y s}-\Gamma^{i d i o}, \\
& A_{110}^{s}=a_{110}^{s} I_{2 \times 2}-e^{-\gamma J_{c}}\left(1-\alpha_{\gamma} J_{c}\right) \Gamma^{s y s}-\Gamma^{i d i o}, \\
& A_{100}^{s}=a_{100}^{s} I_{2 \times 2}-e^{-J_{c}} \Gamma^{s y s}-\Gamma^{i d i o},
\end{aligned}
$$

and

$$
\begin{aligned}
& B_{101}^{s}(\tau) \equiv b_{101}^{s} e^{-\tau A_{111}^{s} \theta^{s}}+\alpha_{\gamma} \beta J_{c} e^{-J_{c}} \Gamma^{s y s} e^{-\tau A_{111}^{s} \theta^{s}}, \\
& B_{110}^{s}(\tau) \equiv b_{110}^{s} e^{-\tau A_{111}^{s} \theta^{s}}-e^{-J_{c}}\left(1-\alpha_{\gamma} J_{c}\right) \alpha_{L} J_{c} \Gamma^{s y s} e^{-\tau A_{111}^{s} \theta^{s}} \text {, }
\end{aligned}
$$

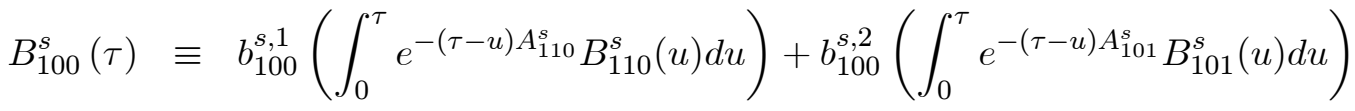

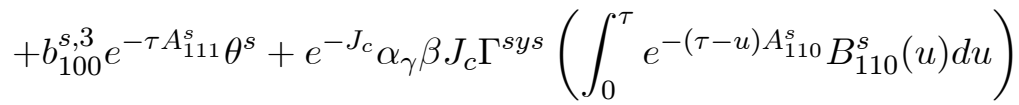

$$
\begin{aligned}
& -e^{-\gamma J_{c}} \alpha_{L} J_{c} \Gamma^{s y s}\left(\int_{0}^{\tau} e^{-(\tau-u) A_{101}^{s}} B_{101}^{s}(u) d u\right)-e^{-J_{c}} \alpha_{\gamma} \alpha_{L} \beta J_{c}^{2} \Gamma^{s y s} e^{-\tau A_{111}^{s} \theta^{s}},
\end{aligned}
$$


where $a_{h}^{s}$ in (A.22) and $b_{h}^{s}$ in (A.23) are given by

$$
\begin{aligned}
& a_{111}^{s}=\rho+\mu-\sigma^{2}+k_{\gamma}-\alpha_{\gamma} J_{c} \lambda_{c}+k_{L}-\alpha_{\gamma} \sigma^{2}-\lambda_{c}\left[e^{-J_{c}}\left(1-\alpha_{\gamma} J_{c}\right)-1\right], \\
& a_{101}^{s}=\rho+\mu-\sigma^{2}+k_{L}-\lambda_{c}\left(e^{-J_{c}}-1\right), \\
& a_{110}^{s}=\rho+\mu-\sigma^{2}+k_{\gamma}-\alpha_{\gamma} J_{c} \lambda_{c}-\alpha_{\gamma} \sigma^{2}-\lambda_{c}\left[e^{-J_{c}}\left(1-\alpha_{\gamma} J_{c}\right)-1\right], \\
& a_{100}^{s}=\rho+\mu-\sigma^{2}-\lambda_{c}\left(e^{-J_{c}}-1\right),
\end{aligned}
$$

and

$$
\begin{aligned}
b_{101}^{s} & =k_{\gamma} \bar{\gamma}-\alpha_{\gamma} \beta J_{c} \lambda_{c}-\alpha_{\gamma} \beta \sigma^{2}+\lambda_{c} e^{-J_{c}} \alpha_{\gamma} \beta J_{c} \\
b_{110}^{s} & =k_{L} \bar{L}+\alpha_{L} J_{c} \lambda_{c}+\tilde{\rho} \alpha_{L} \sigma^{2}+\tilde{\rho} \alpha_{\gamma} \alpha_{L} \sigma^{2}-\lambda_{c} e^{-J_{c}}\left(1-\alpha_{\gamma} J_{c}\right) \alpha_{L} J_{c} \\
b_{100}^{s, 1} & =k_{\gamma} \bar{\gamma}-\alpha_{\gamma} \beta J_{c} \lambda_{c}-\alpha_{\gamma} \beta \sigma^{2}+\lambda_{c} e^{-J_{c}} \alpha_{\gamma} \beta J_{c} \\
b_{100}^{s, 2} & =k_{L} \bar{L}+\alpha_{L} J_{c} \lambda_{c}+\tilde{\rho} \alpha_{L} \sigma^{2}-\lambda_{c} e^{-J_{c}} \alpha_{L} J_{c} \\
b_{100}^{s, 3} & =-\tilde{\rho} \alpha_{\gamma} \alpha_{L} \beta \sigma^{2}-\lambda_{c} e^{-J_{c}} \alpha_{\gamma} \alpha_{L} \beta J_{c}^{2} .
\end{aligned}
$$

The closed form valuation of $\psi^{c}($.$) can be obtained by the same logic. We omit the derivation$ and simply report the result:

$$
\psi^{c}\left(x_{t}, \tau\right) \equiv\left[\psi^{c}\left(x_{t} ; 1\right), \psi^{c}\left(x_{t} ; 2\right)\right]^{\prime}=\eta_{11}^{c}(\tau) x_{1 t} x_{2 t}+\eta_{10}^{c}(\tau) x_{1 t}
$$

where

$$
\begin{aligned}
& \eta_{11}^{c}(\tau)=e^{-\tau A_{11}^{c} \theta^{c}} \\
& \eta_{10}^{c}(\tau)=\int_{0}^{\tau} e^{-(\tau-u) A_{10}^{c} B_{10}^{c}(u) d u} .
\end{aligned}
$$

In (A.27), $A_{11}^{c}, A_{10}^{c}$, and $B_{10}^{c}(\tau)$ are given by

$$
\begin{aligned}
A_{11}^{c} & =a_{11}^{c} I_{2 \times 2}-e^{-J_{c}}\left(1-\alpha_{\gamma} J_{c}\right) \Gamma^{s y s}-\Gamma^{i d i o}, \\
A_{10}^{c} & =a_{10}^{c} I_{2 \times 2}-e^{-J_{c}} \Gamma^{s y s}-\Gamma^{i d i o}, \\
B_{10}^{c}(\tau) & =b_{10}^{c} e^{-\tau A_{11}^{c \prime} \theta^{c}}+\alpha_{\gamma} \beta J_{c} e^{-J_{c}} \Gamma^{s y s} e^{-\tau A_{11}^{c \prime}} \theta^{c},
\end{aligned}
$$

where

$$
\begin{aligned}
& a_{11}^{c}=\rho+\mu-\sigma^{2}+k_{\gamma}-\alpha_{\gamma} J_{c} \lambda_{c}-\alpha_{\gamma} \sigma^{2}-\lambda_{c}\left[e^{-J_{c}}\left(1-\alpha_{\gamma} J_{c}\right)-1\right], \\
& a_{10}^{c}=\rho+\mu-\sigma^{2}-\lambda_{c}\left(e^{-J_{c}}-1\right) \\
& b_{10}^{c}=k_{\gamma} \bar{\gamma}-\alpha_{\gamma} \beta J_{c} \lambda_{c}-\alpha_{\gamma} \beta \sigma^{2}+\lambda_{c} e^{-J_{c}} \alpha_{\gamma} \beta J_{c} .
\end{aligned}
$$


By combining (A.7), (A.16), and (A.26) and using the expressions for $\eta^{s}(\tau)$ and $\eta^{c}(\tau)$, we get

$$
\begin{aligned}
& P_{t, \tau}^{T P} \equiv\left[P_{t, \tau}^{T P}(1), P_{t, \tau}^{T P}(2)\right]^{\prime}=\frac{1}{x_{1 t} x_{2 t}}\left[\psi^{c}\left(x_{t}\right)+\psi^{s}\left(x_{t}\right)\right]
\end{aligned}
$$

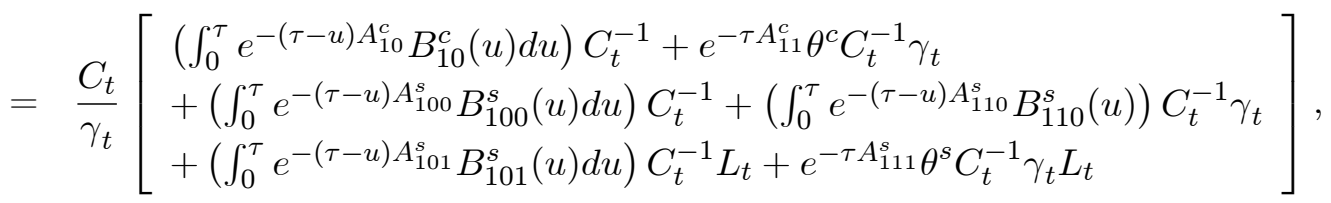

where we have used the definitions of $\left(x_{1 t}, x_{2 t}, x_{3 t}\right)$ given by (A.10). Rearranging yields

$$
P_{t, \tau}^{T P}=\alpha_{1}^{T P}(\tau)+\alpha_{2}^{T P}(\tau) \frac{1}{\gamma_{t}}+\alpha_{3}^{T P}(\tau) L_{t}+\alpha_{4}^{T P}(\tau) \frac{L_{t}}{\gamma_{t}}
$$

where

$$
\begin{aligned}
& \alpha_{1}^{T P}(\tau)=\int_{0}^{\tau} e^{-(\tau-u) A_{110}^{s} B_{110}^{s}(u) d u+e^{-\tau A_{11}^{c}} \theta^{c}} \\
& \alpha_{2}^{T P}(\tau)=\int_{0}^{\tau} e^{-(\tau-u) A_{100}^{s \prime}} B_{100}^{s}(u) d u+\int_{0}^{\tau} e^{-(\tau-u) A_{10}^{c}} B_{10}^{c}(u) d u \\
& \alpha_{3}^{T P}(\tau)=e^{-\tau A_{111}^{s} \theta^{s}} \\
& \alpha_{4}^{T P}(\tau)=\int_{0}^{\tau} e^{-(\tau-u) A_{101}^{s \prime}} B_{101}^{s}(u) d u .
\end{aligned}
$$

The intuition of the above derivation is as follows. Default induces a structural change in the bond payoffs. We thus need to use vector integrations taking into account the interaction between the non-default and the default regime in order to obtain bond prices in both regimes.

\section{A.2 Proof of Proposition 1}

The price of a non-defaultable bond is a special case of a defaultable TP bond where $\lambda^{\text {sys }}=\lambda^{\text {idio }}=$ 0 . In this case Lemma 1 gives

$$
P_{t, \tau}^{0}=\alpha_{1}^{0}(\tau)+\alpha_{2}^{0}(\tau) \tau \frac{1}{\gamma_{t}}
$$

where

$$
\begin{aligned}
& \alpha_{1}^{0}(\tau)=\int_{0}^{\tau} e^{-(\tau-u) A_{110}^{s}} B_{110}^{s}(u) d u+e^{-\tau A_{11}^{c} \theta^{c}} \\
& \alpha_{2}^{0}(\tau)=\int_{0}^{\tau} e^{-(\tau-u) A_{100}^{s \prime} B_{100}^{s}(u) d u+\int_{0}^{\tau} e^{-(\tau-u) A_{10}^{c}} B_{10}^{c}(u) d u}
\end{aligned}
$$

and where $A_{h}$, and $B_{h}^{s}$ are as defined in Lemma 1 with $\lambda^{\text {sys }}$ and $\lambda^{\text {idio }}$ set to zero.

The dynamic process for the instantaneous return on the default-free bond is

$$
\frac{d P_{t, \tau}^{0}}{P_{t, \tau}^{0}}=\mu_{P t}^{0} d t+\sigma_{P t}^{0} d B_{t}+J_{P t}^{0} d N_{t}, \text { where }
$$




$$
\mu_{P t}^{0}=\frac{1}{P_{t, \tau}^{0}}\left[\frac{d \alpha_{1}^{0}(\tau)}{d \tau}+\frac{d \alpha_{1}^{0}(\tau)}{d \tau} \frac{1}{\gamma_{t}}\right]+\alpha_{2}^{0}(\tau) \frac{1}{\gamma_{t} P_{t, \tau}^{0}}\left(-\mu_{\gamma t}+\sigma_{\gamma t}^{2}\right)
$$

and where $\sigma_{P t}^{0}$ and $J_{P t}^{0}$ are given in Proposition 1 . The bond return process can be derived by applying Ito's lemma for jump-diffusions to the bond pricing formula for $P_{t, \tau}^{0}$.

\section{A.3 Proof of Proposition 2}

Proposition 2 states that

$$
P_{t, \tau}^{R F V}=\alpha_{1}^{R F V}(\tau)+\alpha_{2}^{R F V}(\tau) \frac{1}{\gamma_{t}}+\alpha_{3}^{R F V}(\tau) L_{t}+\alpha_{4}^{R F V}(\tau) \frac{L_{t}}{\gamma_{t}}
$$

where we use superscript RFV to denote recovery of face value to distinguish it from the TP case in Lemma 1. We will now show that

$$
\begin{aligned}
& \alpha_{1}^{R F V}(\tau)=e^{-\tau \tilde{A}_{11}^{c}} \theta^{c}+\int_{0}^{\tau} e^{-(\tau-u) \tilde{A}_{110}^{s}} \tilde{B}_{110}^{s}(u) d u, \\
& \alpha_{2}^{R F V}(\tau)=\int_{0}^{\tau} e^{-(\tau-u) \tilde{A}_{10}^{c}} \tilde{B}_{10}^{c}(u) d u+\int_{0}^{\tau} e^{-(\tau-u) \tilde{A}_{100}^{s} \tilde{B}_{100}^{s}(u) d u,} \\
& \alpha_{3}^{R F V}(\tau)=e^{-\tau \tilde{A}_{111}^{s} \theta^{s}}, \\
& \alpha_{4}^{R F V}(\tau)=\int_{0}^{\tau} e^{-(\tau-u) \tilde{A}_{101}^{s} \tilde{B}_{101}^{s}(u) d u} .
\end{aligned}
$$

In the above formula,

$$
\tilde{A}_{h} \equiv\left[\begin{array}{ll}
1 & 0 \\
0 & 0
\end{array}\right] A_{h},
$$

for $h \in\{111,101,110,100,11,10\}$, where $A_{h}$ are given in Lemma 1 . Note we do not differentiate between $A_{h}^{s}$ and $A_{h}^{c}$ in (A.34). This is also the case for $\tilde{B}_{h}(\tau)$ which is defined as follows:

$$
\tilde{B}_{h}(\tau) \equiv\left[\begin{array}{ll}
1 & 0 \\
0 & 0
\end{array}\right] \hat{B}_{h}(\tau)
$$

where $h \in\{101,110,100,10\}$. In (A.35), $\hat{B}_{h}(\tau)$ s are defined by

$$
\begin{aligned}
& \hat{B}_{101}^{s}(\tau) \equiv b_{101}^{s} e^{-\tau \tilde{A}_{111}^{s} \theta^{s}}+\alpha_{\gamma} \beta J_{c} e^{-J_{c}} \Gamma^{s y s} e^{-\tau \tilde{A}_{111}^{s} \theta^{s}}, \\
& \hat{B}_{110}^{s}(\tau) \equiv b_{110}^{s} e^{-\tau \tilde{A}_{111}^{s}} \theta^{s}-e^{-J_{c}}\left(1-\alpha_{\gamma} J_{c}\right) \alpha_{L} J_{c} \Gamma^{s y s} e^{-\tau \tilde{A}_{111}^{s} \theta^{s}} \text {, } \\
& \hat{B}_{100}^{s}(\tau) \equiv b_{100}^{s, 1}\left(\int_{0}^{\tau} e^{-(\tau-u) \tilde{A}_{110}^{s}} \tilde{B}_{101}^{s}(u) d u\right)+b_{100}^{s, 2}\left(\int_{0}^{\tau} e^{-(\tau-u) \tilde{A}_{101}^{s}} \tilde{B}_{101}^{s}(u) d u\right)
\end{aligned}
$$

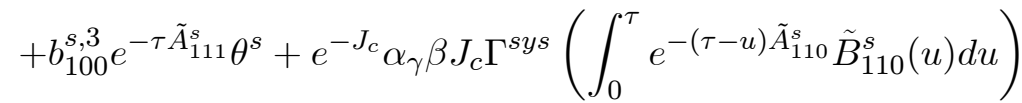

$$
\begin{aligned}
& -e^{-J_{c}} \alpha_{L} J_{c} \Gamma^{s y s}\left(\int_{0}^{\tau} e^{-(\tau-u) \tilde{A}_{101}^{s}} \tilde{B}_{101}^{s}(u) d u\right)-e^{-J_{c}} \alpha_{\gamma} \alpha_{L} \beta J_{c}^{2} \Gamma^{s y s} e^{-\tau \tilde{A}_{111}^{s} \theta^{s},}
\end{aligned}
$$




$$
\hat{B}_{10}^{c}(\tau) \equiv b_{10}^{c} e^{-\tau \tilde{A}_{11}^{c} \theta^{c}}+\alpha_{\gamma} \beta J_{c} e^{-J_{c}} \Gamma^{s y s} e^{-\tau \tilde{A}_{11}^{c}} \theta^{c},
$$

where the $b_{h}$ terms are identical to those given in Lemma 1.

With appropriate revisions of $A$ and $B(\tau)$, the bond price coefficients under RFV are similar to those in Lemma 1. Note that under RFV, bondholders receive the residual payment immediately upon default so that $P_{t, \tau}^{R F V}(2)=1-L_{\tau}$, where $\tau(<T)$ denotes the time of default. Translated into $\psi^{c}($.$) and \psi^{s}($.$) which are defined as in (A.8)-(A.9), we have$

$$
\begin{aligned}
& \psi^{c}\left(x_{\tau}, \tau ; 2\right)=C_{\tau}^{-1} \gamma_{\tau}=x_{1 \tau} x_{2 \tau} \\
& \psi^{s}\left(x_{\tau}, \tau ; 2\right)=-C_{\tau}^{-1} \gamma_{\tau} L_{\tau}=-x_{1 \tau} x_{2 \tau} x_{3 \tau} .
\end{aligned}
$$

Equations (A.37)-(A.38) give defaultable bond prices under RFV conditional on a default regime which is known. Below, we use $\psi^{s}$ (.) as an example to show that the ODEs for the bond price under TP in Lemma 1 can be adjusted to accommodate the stopping time problem in RFV.

We conjecture-and later verify-that the solution for $\psi^{s}($.$) under RFV is still given by (A.16)$ and (A.21). Equation (A.38) thus implies

$$
\eta_{111}^{s}(\tau ; 2)=\theta^{s}(2)=-1, \text { and } \eta_{h}(\tau ; 2)=0 \text { for } h \in\{101,110,100\}
$$

where we have used that $\eta_{111}^{s}$ is the coefficient associated with $x_{1 t} x_{2 t} x_{3 t}$. It is easy to check that (A.39) is the solution to

$$
\frac{d \eta_{h}^{s}(\tau ; 2)}{d \tau}=0, \text { subject to } \eta_{111}^{s}(0 ; 2)=\theta^{s}(2) \text { and } \eta_{h}^{s}(0 ; 2)=0 \text { for } h \neq 111
$$

Recall that under TP $\eta_{h}^{s}(\tau)$ satisfies the ODEs of (A.17) which we rewrite as follows:

$$
\frac{d \eta_{h}^{s}(\tau)}{d \tau}=-A_{h}^{s} \eta_{h}^{s}(\tau)+B_{h}^{s}(\tau) \text { subject to: } \eta_{h}^{s}(0)=\theta_{h}^{s}
$$

where $\theta_{h}^{s}, A_{h}^{s}$, and $B_{h}^{s}(\tau)$ are given by (A.18), (A.19), and (A.20). Below, we use a 3 -step procedure to show that (A.40) can be accommodated by (A.41) through appropriate modifications of $A_{h}^{s}$ and $B_{h}^{s}(\tau)$.

- Step 1: We go from $A_{h}$ to $\tilde{A}_{h}$ by setting the last row of $A_{h}$ to zeros so that the last row of $-A_{h} \eta_{h}(\tau)$ also is zero. This step provides (A.34).

- Step 2: Note that the values of $B_{h}(\tau) s$ are derived from the $A_{h}$ s and hence cannot be directly modified. Instead, we replace $A_{h}$ with $\tilde{A}_{h}$ and then compute the implied $B$ s using (A.23) which we have denoted by $\hat{B}_{h}(\tau)$. This step ensures that the structure of ODE for $\eta_{h}(\tau, 1)$ which determines the pricing conditional on non-default regime is unaffected, and it provides (A.36). 
- Step 3: We set the last entry of $\hat{B}_{h}(\tau)$ to zero so as to fulfill the requirement in (A.40). The resulting $\tilde{B}_{h}(\tau)$ provides (A.35).

It is worth pointing out that when calculating $\hat{B}_{100}^{s}(\tau)$ in the second step, we use $\left(\tilde{B}_{101}^{s}, \tilde{B}_{110}^{s}\right)$ instead of $\left(\hat{B}_{101}^{s}, \hat{B}_{110}^{s}\right)$. This is because the $B_{h}$ matrices under this scenario are computed by backward induction starting from $B_{111}=0_{2 \times 1}$. For example, in order to calculate $B_{100}^{s}(\tau)$ under TP, we need to use the previously computed $B_{101}^{s}$ and $B_{110}^{s}$. Similarly, in order to calculate $\tilde{B}_{100}^{s}(\tau)$ via $\hat{B}_{100}^{s}(\tau)$ under $\mathrm{RFV}$, we need to use the previously computed $\tilde{B}_{101}^{s}$ and $\tilde{B}_{110}^{s}$.

\section{A.4 Closed-form Expressions for Integrals}

At this stage, we have completed the proof of the solution for the defaultable bond under the RFV assumption. However, the pricing formulas in both Lemma 1 and Proposition 2 are presented up to a set of integral computations. On the one hand, the vector integrations can be easily computed by methods of Gaussian quadrature (e.g., Miranda and Fackler, 2002), given that integrands are in closed forms. However, we have also derived complete closed-form solutions for the expressions for $P_{t, \tau}^{T P}$ and $P_{t, \tau}^{R F V}$ by analytically evaluating the vector integrations appearing in the above proofs of Lemma 1 and Proposition 2.

To save space, we only report the analytical expressions of the $\alpha$-coefficients in (A.33), and the details of derivations are available upon request. To facilitate the presentation, we first define

$$
\begin{aligned}
c_{11} & \equiv e^{-J_{c}}\left(1-\alpha_{\gamma} J_{c}\right) \lambda^{s}+\lambda^{i d i o}, \\
c_{10} & \equiv e^{-J_{c}} \lambda^{\text {sys }}+\lambda^{i d i o} .
\end{aligned}
$$

Given (A.42), we have

$$
\begin{aligned}
& \alpha_{1}^{R F V}=\left[\begin{array}{c}
\frac{a_{11}^{c}}{a_{11}^{c}+c_{11}} e^{-\tau\left(a_{11}^{c}+c_{11}\right)}+\frac{c_{11}}{a_{11}^{c}+c_{11}} \\
1
\end{array}\right]-b_{110}^{s}\left(\frac{c_{11}}{a_{111}^{s}+c_{11}}\right) e^{-\tau\left(a_{110}^{s}+c_{11}\right)} . \\
& {\left[\begin{array}{c}
\frac{e^{-\tau\left(a_{111}^{s}-a_{110}^{s}\right)}-1}{a_{111}^{s}-a_{110}^{s}}+\frac{e^{\tau\left(a_{110}^{s}+c_{11}\right)}-1}{a_{110}^{s}+c_{11}} \\
0
\end{array}\right]} \\
& +e^{-J_{c}}\left(1-\alpha_{\gamma} J_{c}\right) \alpha_{L} J_{c} e^{-\tau\left(a_{110}^{s}+c_{11}\right)} \lambda^{s y s}\left[\begin{array}{c}
-\frac{c_{11}}{a_{111}^{s}+c_{11}} \frac{e^{-\tau\left(a_{11}^{s}-a_{110}^{s}\right)}-1}{a_{111}^{s}-a_{110}^{s}} \\
+\frac{a_{111}^{s}}{a_{111}^{s}+c_{11}} \frac{e^{\tau\left(a_{110}^{s}+c_{11}\right)}-1}{a_{110}^{s}+c_{11}} \\
0
\end{array}\right] \text {, } \\
& \alpha_{3}^{R F V}=\left[\begin{array}{c}
\left(\frac{c_{11}}{a_{111}^{s}+c_{11}}\right)\left(e^{-\tau\left(a_{111}^{s}+c_{11}\right)}-1\right) \\
-1
\end{array}\right] \\
& \alpha_{4}^{R F V}=-b_{100}^{s} \frac{c_{11}}{a_{111}^{s}+c_{11}} e^{-\tau\left(a_{101}^{s}+c_{11}\right)}\left[\begin{array}{c}
\frac{e^{-\tau\left(a_{111}^{s}-a_{101}^{s}\right)}-1}{a_{111}^{s}-a_{101}^{s}}+\frac{e^{\tau\left(a_{101}^{s}+c_{11}\right)}-1}{a_{101}^{s}+c_{11}} \\
0
\end{array}\right]
\end{aligned}
$$




$$
-\alpha \beta J_{c} e^{-J_{c}} e^{-\tau\left(a_{101}^{s}+c_{11}\right)} \lambda^{s y s}\left[\begin{array}{c}
-\frac{c_{11}}{a_{111}^{s}+c_{11}} \frac{e^{-\tau\left(a_{11}^{s}-a_{101}^{s}\right)}-1}{a_{111}^{s}-a_{101}^{s}} \\
+\frac{a_{111}^{s}}{a_{111}^{s}+c_{11}} \frac{e^{\tau\left(a_{101}^{s}+c_{11}\right)}-1}{a_{101}^{s}+c_{11}} \\
0
\end{array}\right]
$$

where the $a$ s and $b$ s are given by (A.24), (A.25) and (A.29). We can also derive,

$\alpha_{2}^{R F V}(\tau)=\left[\begin{array}{c}\Theta_{0}(\tau) \\ 0\end{array}\right]+\left(b_{100}^{s, 1}-e^{-J_{c}} \alpha_{\gamma} \beta J_{c} \lambda^{s y s}\right)\left[\begin{array}{c}\Theta_{1}(\tau) \\ 0\end{array}\right]+\left(b_{100}^{s, 2}+e^{-J_{c}} \alpha_{L} J_{c} \Gamma^{s y s} \lambda^{s y s}\right)\left[\begin{array}{c}\Theta_{2}(\tau) \\ 0\end{array}\right]$,

where we in addition have defined:

$$
\begin{aligned}
& \Theta_{0}(\tau) \equiv e^{-\tau\left(a_{11}^{c}+c_{10}\right)}\left(-\frac{c_{11}}{a_{111}^{s}+c_{11}} \frac{e^{-\tau\left(a_{11}^{c}+c_{11}-a_{10}^{c}-c_{10}\right)}-1}{a_{11}^{c}+c_{11}-a_{10}^{c}-c_{10}}+\frac{c_{11}}{a_{11}^{c}+c_{11}} \frac{e^{\tau\left(a_{10}^{c}+c_{10}\right)}-1}{a_{10}^{c}+c_{10}}\right) \\
& -\frac{c_{11}}{a_{11}^{c}+c_{11}} \lambda^{s y s} e^{-\tau\left(a_{11}^{c}+c_{10}\right)}\left[\frac{e^{\tau\left(a_{10}^{c}+c_{10}\right)}-1}{a_{10}^{c}+c_{10}}+\frac{e^{-\tau\left(a_{11}^{c}+c_{11}-a_{10}^{c}-c_{10}\right)}-1}{a_{11}^{c}+c_{11}-a_{10}^{c}-c_{10}}\right] \\
& -b_{100}^{s, 3} \frac{c_{11}}{a_{111}^{s}+c_{11}}\left(\frac{e^{-\tau\left(a_{111}^{s}+c_{11}\right)}-1}{a_{111}^{s}+c_{11}}+\tau\right) \\
& +e^{-J_{c}} \alpha_{\gamma} \alpha_{L} \beta J_{c}^{2} \lambda^{s y s}\left(\frac{c_{11}}{a_{111}^{s}+c_{11}} \frac{e^{-\tau\left(a_{111}^{s}+c_{11}\right)}-1}{a_{111}^{s}+c_{11}}+\frac{a_{111}^{s}}{a_{111}^{s}+c_{11}} \tau\right) \\
& \Theta_{1}(\tau) \equiv-b_{110}^{s} \frac{c_{11}}{a_{111}^{s}+c_{11}} \frac{1}{a_{111}^{s}-a_{110}^{s}}\left[\frac{e^{-\tau\left(a_{110}^{s}+c_{11}\right)}}{a_{110}^{s}+c_{11}}-\frac{e^{-\tau\left(a_{111}^{s}+c_{11}\right)}}{a_{111}^{s}+c_{11}}\right] \\
& -b_{110}^{s} \frac{c_{11}}{a_{111}^{s}+c_{11}} \frac{1}{a_{110}^{s}+c_{11}}\left(\tau+\frac{e^{-\tau\left(a_{110}^{s}+c_{11}\right)}-1}{a_{110}^{s}+c_{11}}\right)+e^{-J_{c}}\left(1-\alpha_{\gamma} J_{c}\right) \alpha_{L} J_{c} e^{-\tau\left(a_{110}^{s}+c_{11}\right)} \lambda^{s y s} . \\
& {\left[\frac{c_{11}}{a_{111}^{s}+c_{11}} \frac{1}{a_{111}^{s}-a_{110}^{s}}\left[\frac{e^{-\tau\left(a_{11}^{s}-a_{110}^{s}\right)}-1}{a_{111}^{s}-a_{110}^{s}}+\tau\right]+\frac{a_{111}^{s}}{a_{111}^{s}+c_{11}} \frac{1}{a_{110}^{s}+c_{11}}\left(\frac{e^{\tau\left(a_{110}^{s}+c_{11}\right)}-1}{a_{110}^{s}+c_{11}}-\tau\right)\right]} \\
& \Theta_{2}(\tau) \equiv-b_{100}^{s} \frac{c_{11}}{a_{111}^{s}+c_{11}} \frac{1}{a_{111}^{s}-a_{101}^{s}}\left[\frac{e^{-\tau\left(a_{111}^{s}\right)}}{a_{111}^{s}+c_{11}}-\frac{e^{-\tau\left(a_{101}^{s}+c_{11}\right)}}{a_{101}^{s}+c_{11}}\right] \\
& -b_{110}^{s} \frac{c_{11}}{a_{111}^{s}+c_{11}} \frac{1}{a_{110}^{s}+c_{11}}\left(\tau+\frac{e^{-\tau\left(a_{101}^{s}+c_{11}\right)}-1}{a_{101}^{s}+c_{11}}\right)-\alpha_{\gamma} \beta J_{c} e^{-J_{c}} e^{-\tau\left(a_{101}^{s}+c_{11}\right)} \lambda^{s y s} . \\
& {\left[\frac{c_{11}}{a_{111}^{s}+c_{11}} \frac{1}{a_{111}^{s}-a_{101}^{s}}\left[\frac{e^{-\tau\left(a_{11}^{s}-a_{101}^{s}\right)}-1}{a_{111}^{s}-a_{101}^{s}}+\tau\right]+\frac{a_{111}^{s}}{a_{111}^{s}+c_{11}} \frac{1}{a_{101}^{s}+c_{11}}\left(\frac{e^{\tau\left(a_{101}^{s}+c_{11}\right)}-1}{a_{101}^{s}+c_{11}}-\tau\right)\right] \text {. }}
\end{aligned}
$$

\section{A.5 Proof of Proposition 3}

In the RFV case we have the bond price formula

$$
P_{t, \tau}^{R F V}=\alpha_{1}^{R F V}(\tau)+\alpha_{2}^{R F V}(\tau) \frac{1}{\gamma_{t}}+\alpha_{3}^{R F V}(\tau) L_{t}+\alpha_{4}^{R F V}(\tau) \frac{L_{t}}{\gamma_{t}}
$$


Applying Ito's lemma for jump-diffusions, we get

$$
\frac{d P_{t, \tau}^{R F V}}{P_{t, \tau}^{R F V}}=\mu_{P t}^{R F V} d t+\sigma_{P t}^{R F V} d B_{t}+J_{P t}^{R F V} d N_{t}^{\perp}+\left[\frac{1-L_{t}}{P_{t^{-}, \tau}^{R F V}}-1\right] d N_{t}^{s y s},
$$

where

$$
\begin{aligned}
\mu_{P t}^{R F V}= & -\frac{1}{P_{t, \tau}^{R F V}}\left[\frac{d \alpha_{1}^{R F V}(\tau)}{d \tau}+\frac{d \alpha_{2}^{R F V}(\tau)}{d \tau} \frac{1}{\gamma_{t}}+\frac{d \alpha_{3}^{R F V}(\tau)}{d \tau} L_{t}+\frac{d \alpha_{4}^{R F V}(\tau)}{d \tau} \frac{L_{t}}{\gamma_{t}}\right] \\
& +\frac{1}{P_{t, \tau}^{R F V}}\left[\alpha_{2}^{R F V}(\tau)+\alpha_{4}^{R F V}(\tau) L_{t}\right] \frac{1}{\gamma_{t}}\left(-\mu_{\gamma t}+\sigma_{\gamma t}^{2}\right) \\
& +\frac{1}{P_{t, \tau}^{R F V}}\left[\alpha_{3}^{R F V}(\tau)+\alpha_{4}^{R F V}(\tau) \frac{1}{\gamma_{t}}\right] k_{L}\left(\bar{L}-L_{t}\right)+\alpha_{L} J_{c} \lambda_{c} \\
& +\frac{1}{P_{t, \tau}^{R F V}} \alpha_{4}^{b, R F V}(\tau) \frac{1}{\gamma_{t}} \sigma_{\gamma t} \alpha_{L} \sigma .
\end{aligned}
$$

The expressions for $\sigma_{P t}^{R F V}$ and $J_{P t}^{R F V}$ are provided in Proposition 3. 


\section{References}

Acharya, V., S. Bharath and A. Srinivasan, 2007. Does industry-wide distress affect defaulted firms? Evidence from creditor recoveries. Journal of Financial Economics 85, 787-821.

Altman, E. and V. Kishore, 1996. Almost everything you wanted to know about recoveries on defaulted bonds. Financial Analysts Journal 52, 57-64.

Bakshi, G., D. Madan, and F. Zhang, 2006. Investigating the role of systematic and firm-specific factors in default risk: Lessons from empirically evaluating credit risk models. Journal of Business $79,1955-1988$.

Barsky, R., M. Kimball, F. Juster, and M. Shapiro, 1997. Preference parameters and behavioral heterogeneity: An experimental approach in the health and retirement survey. Quarterly Journal of Economics 112, 537-579.

Barro, R., 2006. Rare disasters and asset markets in the twentieth century. Quarterly Journal of Economics 121, 822-866.

Barro, R., 2009. Rare disasters, asset prices, and welfare costs. American Economic Review 99, $243-264$.

Barro, R., and J. Ursua, 2008. Macroeconomic crises since 1870. Brookings Papers on Economic Activity 39, Spring, 255-335.

Benzoni L., P. Collin-Dufresne, and R. Goldstein, 2011. Explaining asset pricing puzzles associated with the 1987 market crash. Journal of Financial Economics 101, 552-573.

Berndt, A., R. Douglas, D. Duffie, M. Ferguson, and D. Schranz, 2008. Measuring default risk premia from default swap rates and EDFs. Working paper, Stanford University.

Bhamra, H., L. Kuehn, and I. Strebulaev, 2010. The levered equity risk premium and credit spreads: A unified framework. Review of Financial Studies 23, 645-703.

Blanco, R., S. Brennan, and I. March, 2005. An empirical analysis of the dynamic relationship between investment-grade bonds and credit default swaps. Journal of Finance 60, 2255-2281.

Campbell, J., and J. Cochrane, 1999. By force of habit: A consumption-based explanation of aggregate stock market behavior. Journal of Political Economy 107, 205-251.

Cao, C., F. Yu and Z. Zhong, 2010. The information content of option-implied volatility for credit default swap valuation. Journal of Financial Markets 13, 321-343.

Carr, P and L. Wu, 2011. A simple robust link between American puts and credit protection. Review of Financial Studies 24, 473-505. 
Chen, H., 2010. Macroeconomic conditions and the puzzles of credit spreads and capital structure. Journal of Finance 65, 2171-2212.

Chen, H., S. Joslin, and N. Tran, 2012. Rare disasters and risk sharing with heterogeneous beliefs. Review of Financial Studies 25, 2189-2224.

Chen, L., P. Collin-Dufresne, and R. S. Goldstein, 2009. On the relation between credit spread puzzles and the equity premium puzzle. Review of Financial Studies 22, 3367-3409.

Chen, L., D. Lesmond, and J. Wei, 2007. Corporate yield spreads and bond liquidity. Journal of Finance 62, 119-149.

Chen, N. and S. Kou, 2009. Credit spreads, optimal capital structure, and implied volatility with endogenous default and jump risk. Mathematical Finance 19, 343-378.

Collin-Dufresne, P., R Goldstein, and S. Martin, 2001. The determinants of credit spread changes. Journal of Finance 56, 2177-2207.

Constantinides, G., 2008. Macroeconomic crises since 1870. Comment on Barro and Ursua. Brookings Papers on Economic Activity 39, Spring, 341-350.

Coval, J., J. Jurek, and E. Stafford, 2009. Economic catastrophe bonds. American Economic Review $99,628-666$.

Cremers, M., J. Driessen, and P. Maenhout, 2008. Explaining the level of credit spreads: Optionimplied jump risk premia in a firm value model. Review of Financial Studies 21, 2209-2242.

Cremers, M., J. Driessen, P. Maenhout, and D. Weinbaum, 2008. Individual stock-price implied volatility and credit spreads. Journal of Banking and Finance 32, 2706-2715

Donaldson, J. and R. Mehra, 2008. Risk-based explanations of the equity premium, in J. Donaldson and R. Mehra (eds.), Handbook of the Equity Risk Premium. Elsevier, San Diego.

Driessen, J., 2005. Is default event risk priced in corporate bonds? Review of Financial Studies 18, 165-195.

Du, D., 2011. General equilibrium pricing of options with habit formation and event risks. Journal of Financial Economics 99, 400-426.

Dumas, B., J. Fleming, and R. Whaley, 1998. Implied volatility functions: Empirical tests. Journal of Finance 53, 2059-2106.

Duffie, D., 2001. Dynamic asset pricing theory. Third edition. Princeton University Press, Princeton, NJ. 
Duffie, D., and D. Lando, 2001. Term structure of credit spreads with incomplete accounting information. Econometrica 69, 633-664.

Duffie, D., and K. Singleton, 1999. Modeling term structures of defaultable bonds. Review of Financial Studies 12, 687-720.

Elton, E. J., M. Gruber, D. Agrawal, and C. Mann, 2001. Explaining the rate spread on corporate bonds. Journal of Finance 56, 247-277.

Ericsson, J , and O. Renault, 2006. Liquidity and credit risk. Journal of Finance 61, 2219-2250.

Gabaix, X., 2012. Variable rare disasters: An exactly solved framework for ten puzzles in macrofinance. Quarterly Journal of Economics 127, 645-700.

Gilchrist, S., and E. Zakrajšek, 2012. Credit spreads and business cycle fluctuations. American Economic Review 102, 1692-1720.

Gourio, F., 2008. Disasters and recoveries. American Economic Review, Papers and Proceedings, $98,68-73$.

Gourio, F., 2012. Credit risk and disaster risk. American Economic Journal: Macroeconomics. Forthcoming.

He, Z., and W. Xiong, 2012. Rollover risk and credit risk. Journal of Finance 67, 391-429.

Huang, J., and M. Huang, 2012. How much of the corporate-treasury yield spread is due to credit risk? Review of Asset Pricing Studies 2, 153-202.

Jarrow, R., D. Lando, and S. M. Turnbull, 1997. A Markov model for the term structure of credit risk spreads. Review of Financial Studies 10, 481-523.

Longstaff, F., S. Mithal, and E. Neis, 2005. Corporate yield spreads: Default risk or liquidity? New evidence from the credit default swap market. Journal of Finance 60, 2213-2253.

Mao, X., and C. Yuan, 2006. Stochastic differential equations with Markovian switching. Imperial College Press, London, UK.

Menzly L., Santos, T., and P. Veronesi, 2004. Understanding predictability. Journal of Political Economy 112, 1-47.

Miranda M., and P. Fackler, 2002. Applied computational economics and finance. MIT Press, Cambridge, MA.

Nakamura, E., J. Steinsson, R. Barro, and J. Ursua, 2011. Crises and recoveries in an empirical model of consumption disasters, American Economic Journal: Macroeconomics, forthcoming. 
Pan, J., 2002. The jump-risk premia implicit in options: Evidence from an integrated time-series study. Journal of Financial Economics 63, 3-50.

Rietz, T., 1988. The equity risk premium: A solution. Journal of Monetary Economics 22, 117-131.

Santos, T., and P. Veronesi, 2010. Habit formation, the cross section of stock returns and the cash-flow risk puzzle. Journal of Financial Economics 98, 385-413.

Shleifer, A., and R. Vishny, 1992. Liquidation values and debt capacity. Journal of Finance 47, $1343-1366$.

Wachter, J., 2006. A consumption-based model of the term structure of interest rates. Journal of Financial Economics 79, 365-399.

Wachter, J., 2012. Can time-varying risk of rare disasters explain aggregate stock market volatility? Journal of Finance, forthcoming.

Wang, H., H. Zhou and Y. Zhou 2011. Credit default swap spreads and variance risk premia. Working paper, Federal Reserve Board.

Zhang, B. Y., H. Zhou, and H. Zhu, 2009. Explaining credit default swap spreads with the equity volatility and jump risks of individual firms. Review of Financial Studies 22, 5099-5131. 
Figure 1. Simulated 120-Year Path of the Model Economy
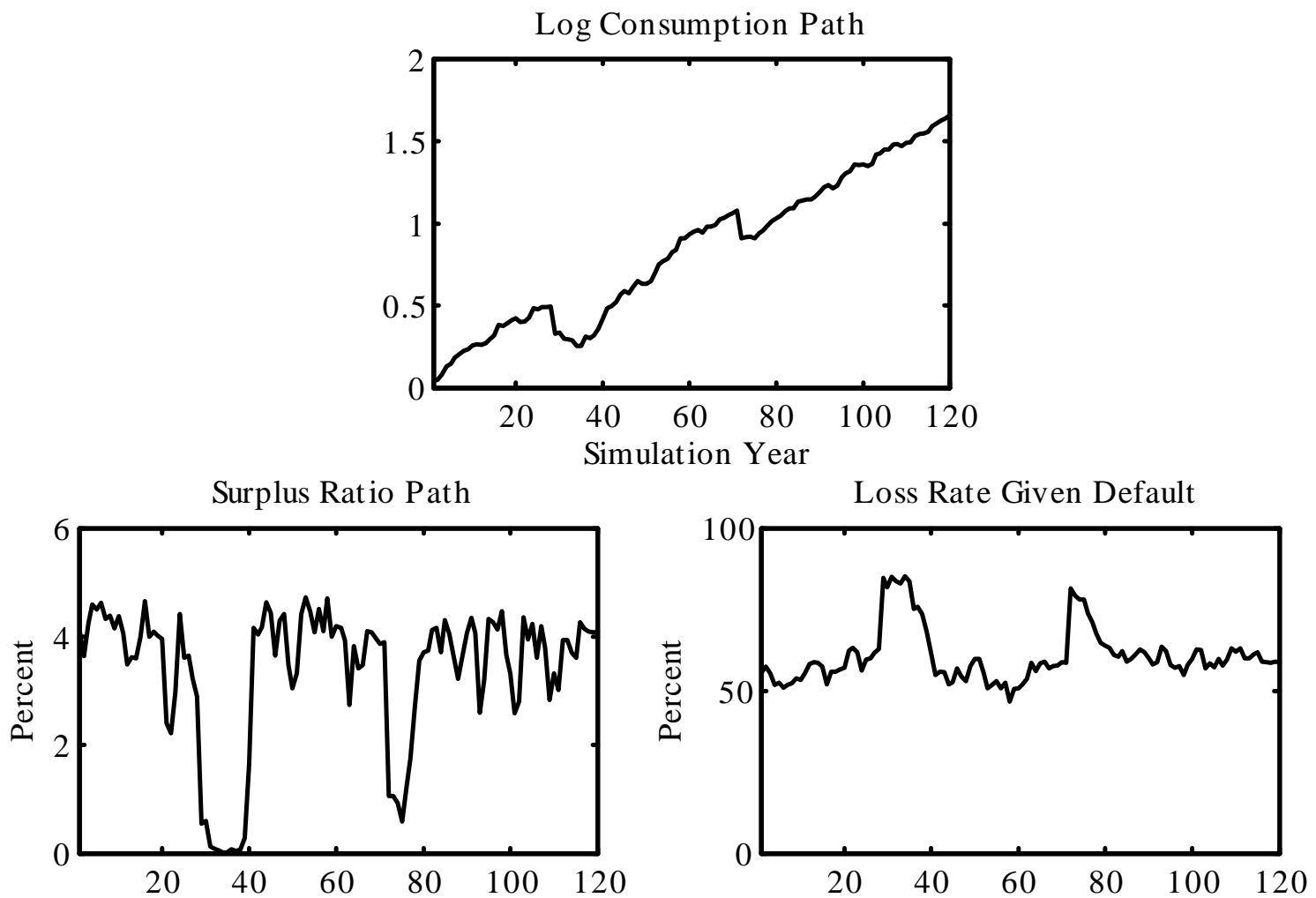

Defaults in Population of 1,000 Aaa Firms

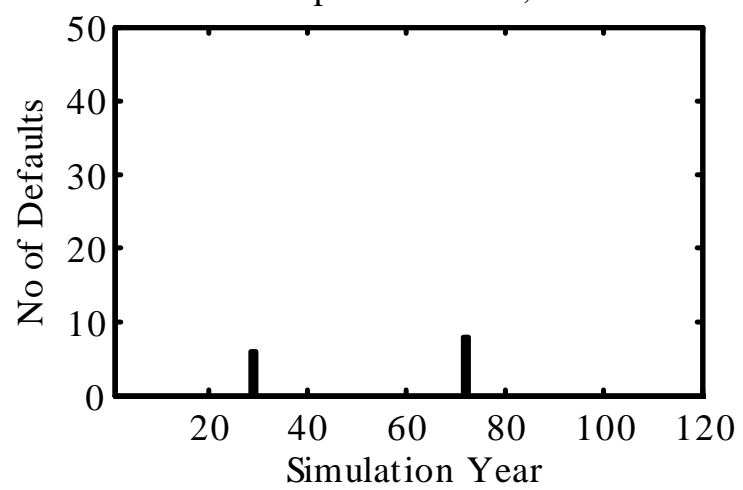

Defaults in Population of 1,000 Baa Firms

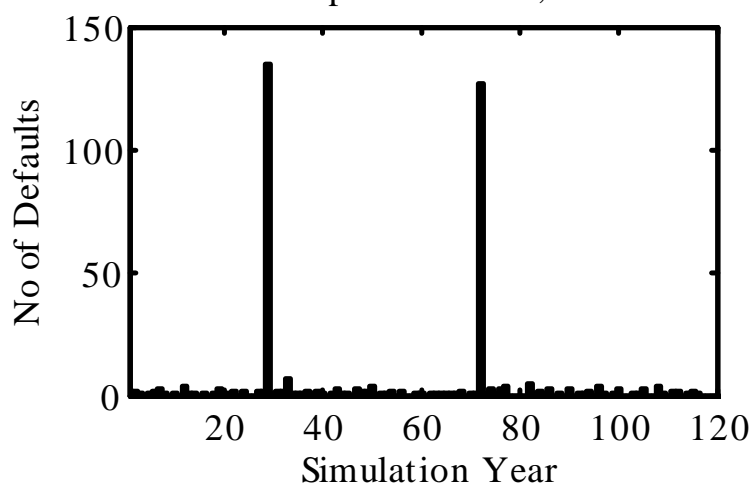

Notes to Figure: We simulate a 120-year path of the model economy. Moderate macro consumption disasters strike at around year 30 and 70 in the particular sample path plotted. 
Figure 2. Model-Implied 5-Year Credit Spreads Plotted Against the Surplus Ratio

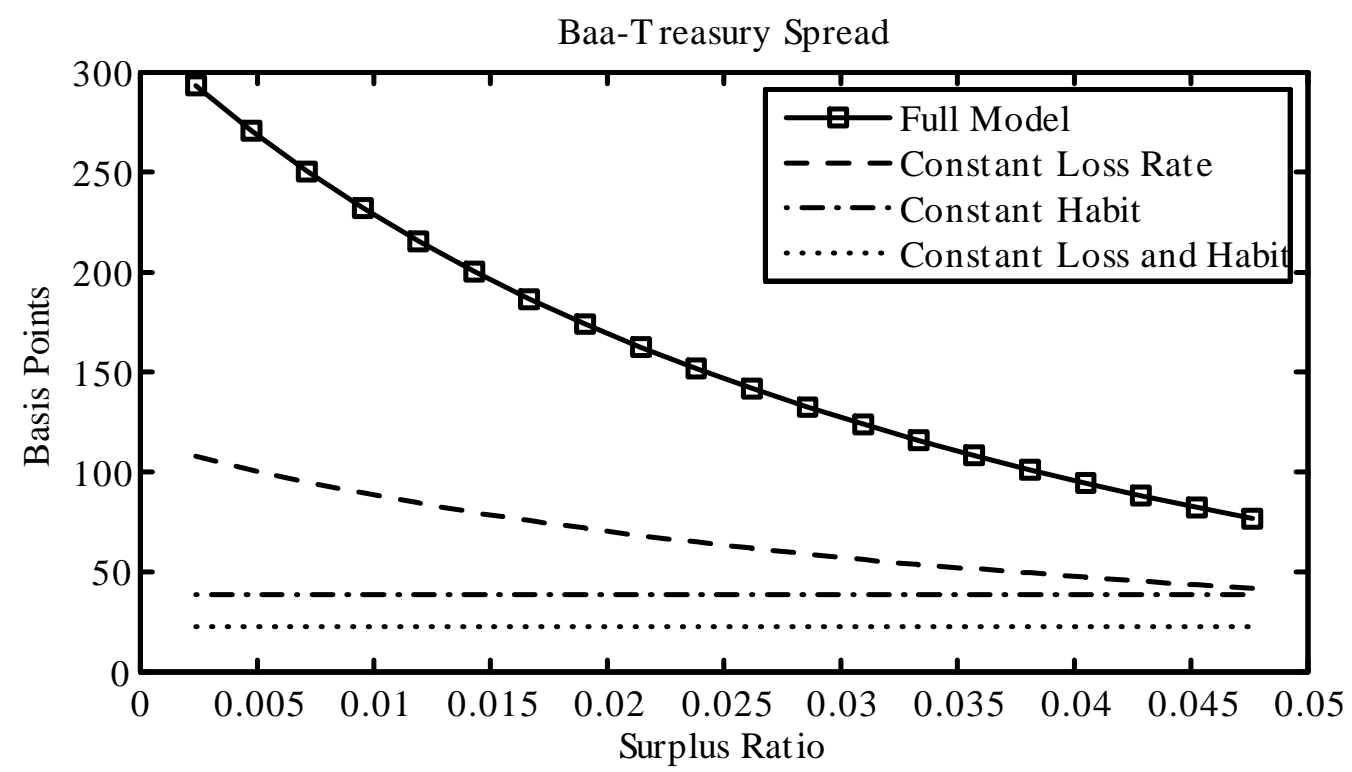

Aaa-Treasury Spread

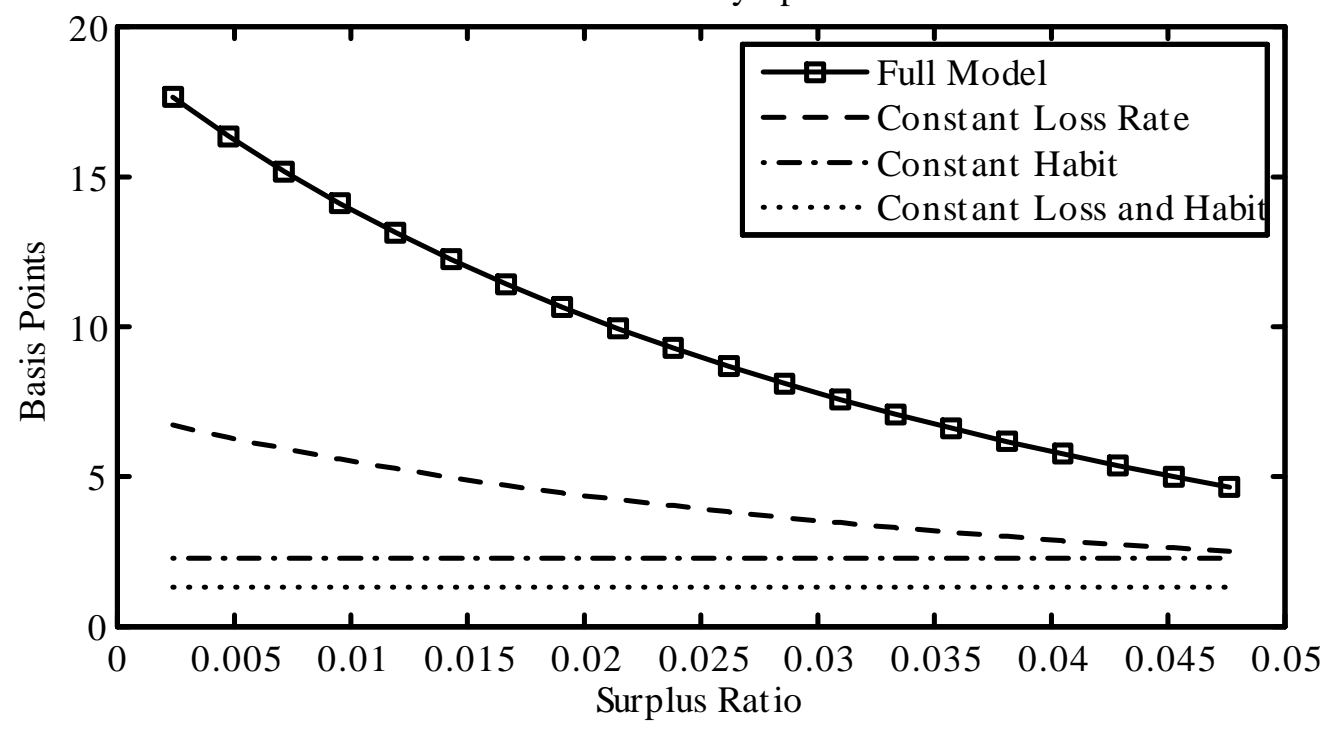

Notes to Figure: In the "Constant Loss and Habit" special case we set $k_{\gamma}=\alpha_{\gamma}=k_{L}=\alpha_{L}=0$. In the "Constant Habit" special case we allow for only variations in $L_{t}$ by setting $k_{\gamma}=\alpha_{\gamma}=0$. In the "Constant Loss Rate" special case we allow for only variations in $\gamma$ by setting $k_{L}=\alpha_{L}=0$. In the "Full Model" $\gamma$ and $L$ are both dynamic. The top panel plot the 5-year Baa-treasury spread as a function of surplus ratio $S$. The bottom two panel shows the 5-year Aaa-Treasury spreads. 
Figure 3. Credit Spreads and Premium Differences versus the Surplus Ratio: Full Model.

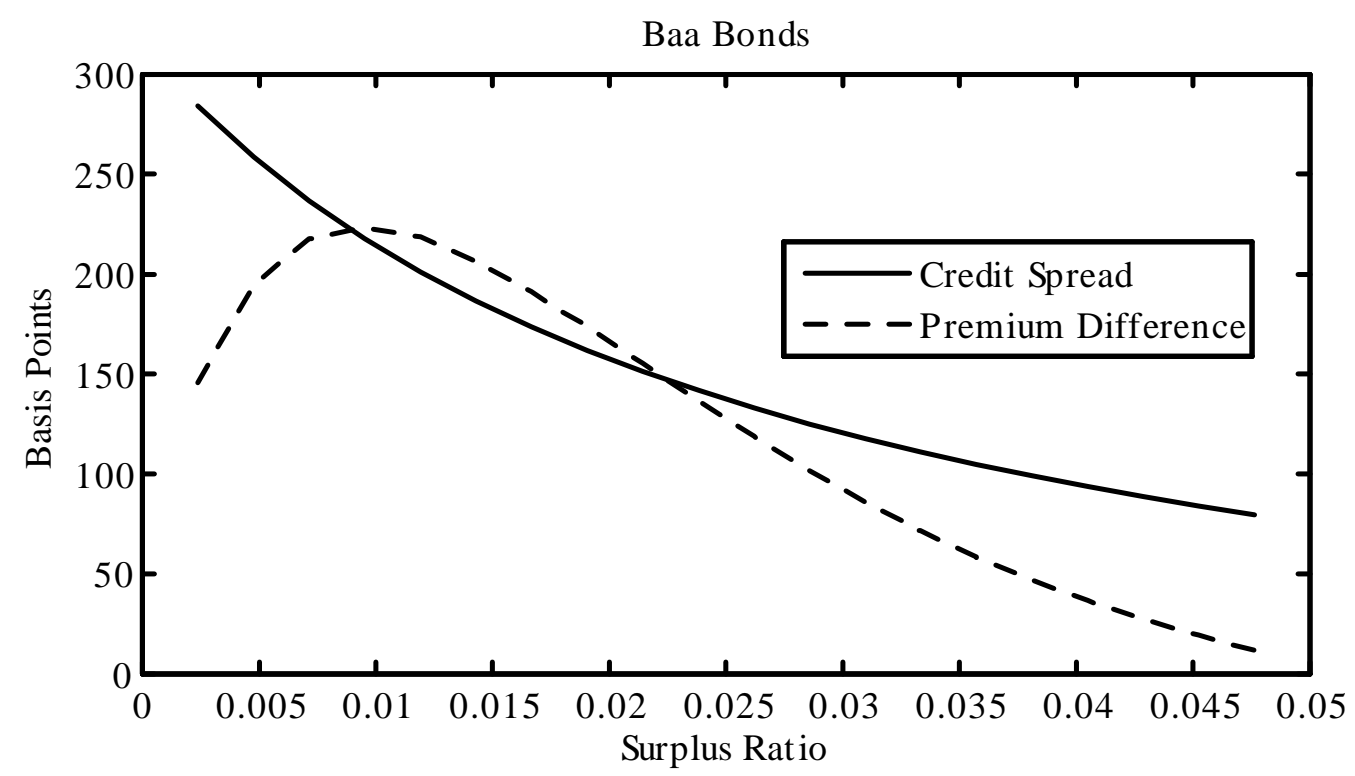

Aaa Bonds

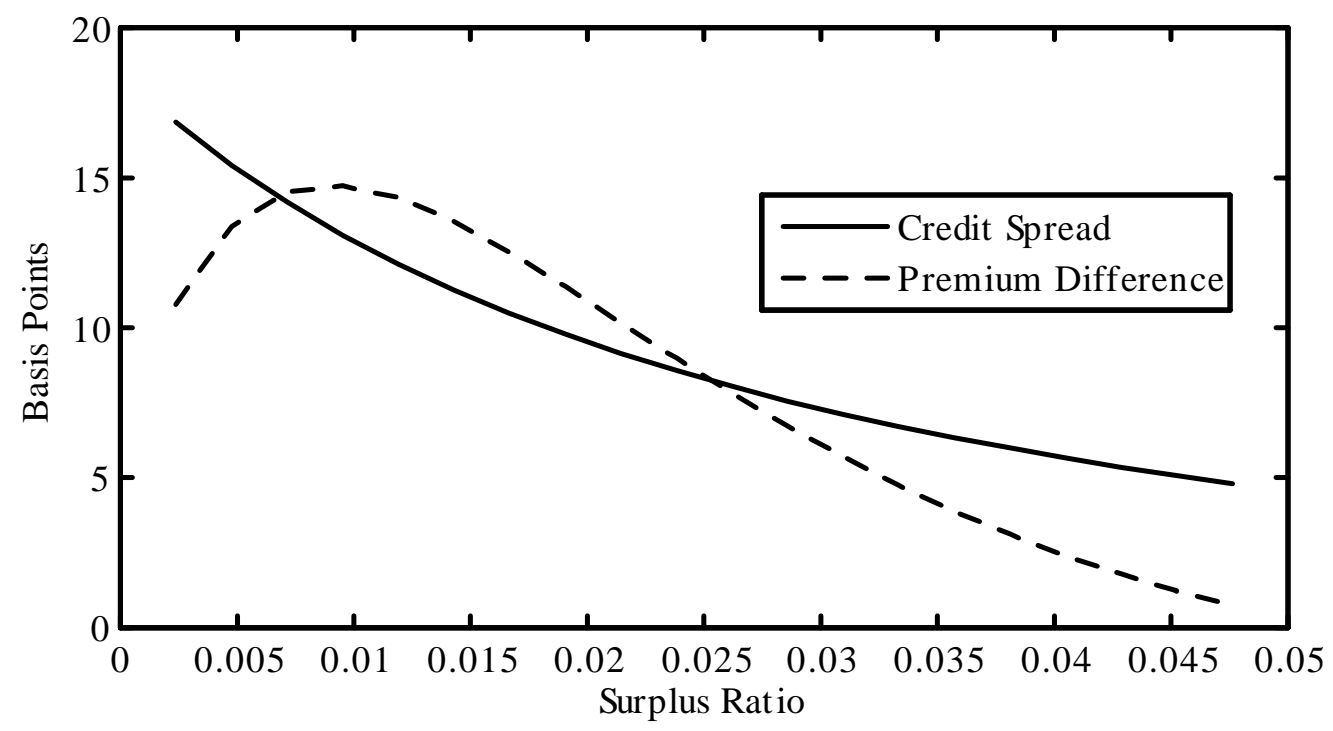

Notes to Figure: We plot the model-based credit spreads (solid lines) and premium differences (dashed lines) in Baa over Treasury (top panel) and in Aaa over Treasury (bottom panel). The surplus ratio, $S$ is on the horizontal axis. The spreads are computed using the full model with dynamic loss rate and habit. 
Figure 4. Term structure of the Baa-Aaa Spread: Market and Models

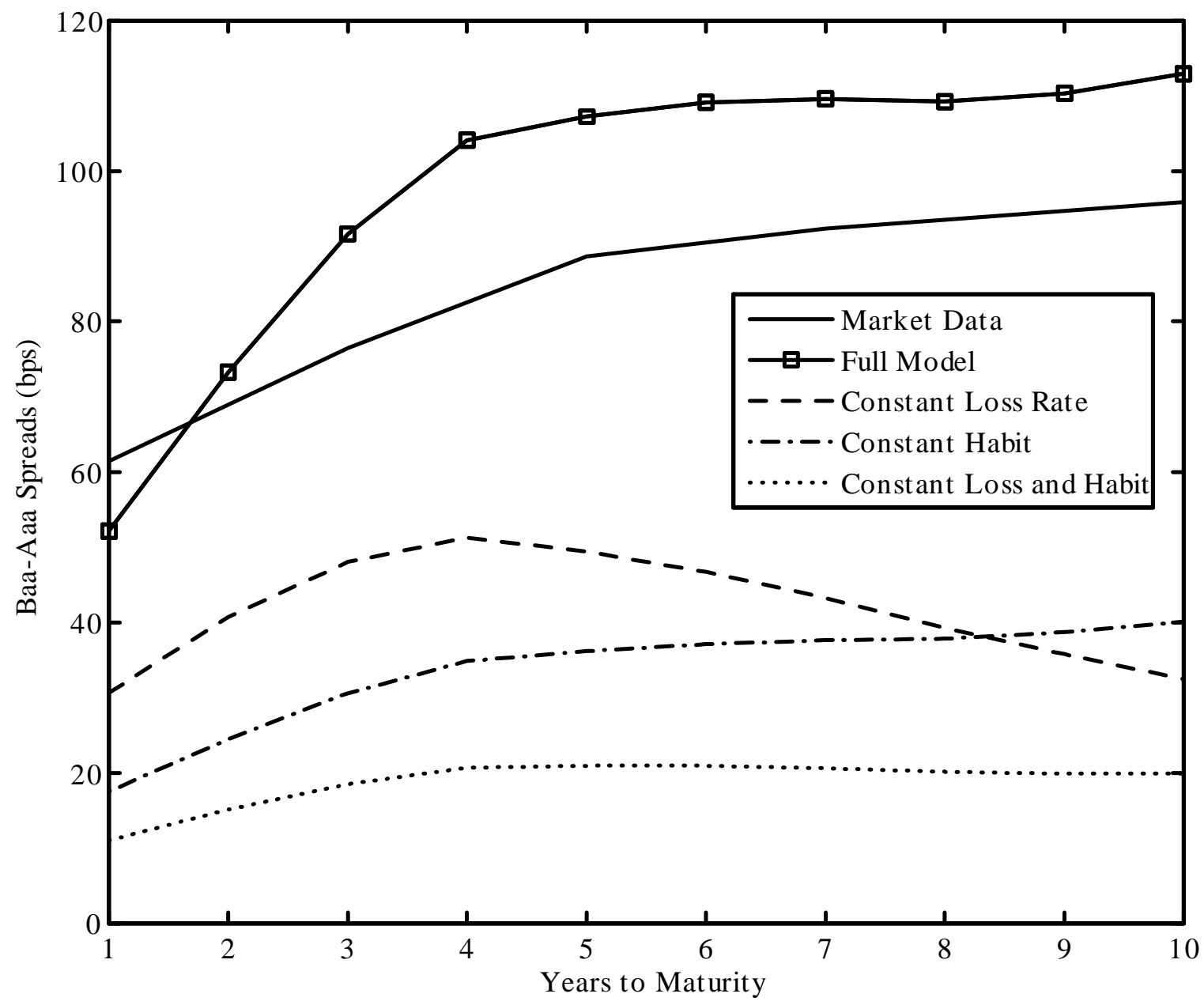

Notes to Figure: The solid line shows the term structure of empirical Baa-Aaa credit spreads for maturities from 1 through 10 years and the other lines show model-implied spreads. In the "Constant Loss and Habit" special case we set $k_{\gamma}=\alpha_{\gamma}=k_{L}=\alpha_{L}=0$. In the "Constant Habit" special case we allow for only variations in $L_{t}$ by setting $k_{\gamma}=\alpha_{\gamma}=0$. In the "Constant Loss Rate" special case we allow for only variations in $\gamma$ by setting $k_{L}=\alpha_{L}=0$. In the "Full Model" $\gamma$ and $L$ are both dynamic. 
Figure 5. Time Series of Credit Spread Levels: Market and Models
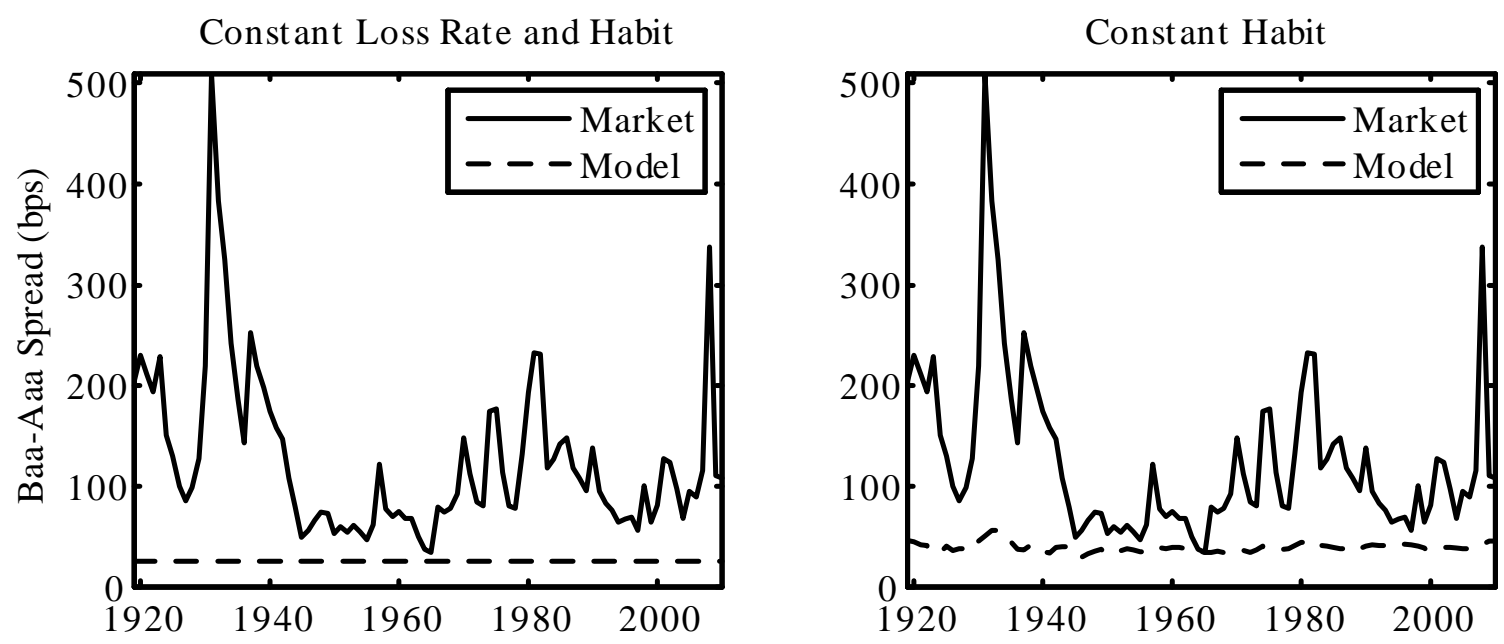

Constant Loss Rate

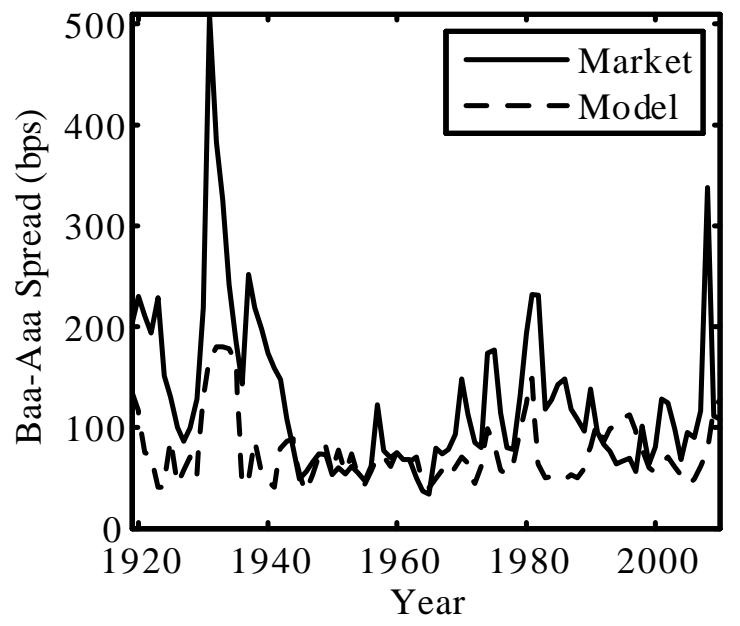

Full Model

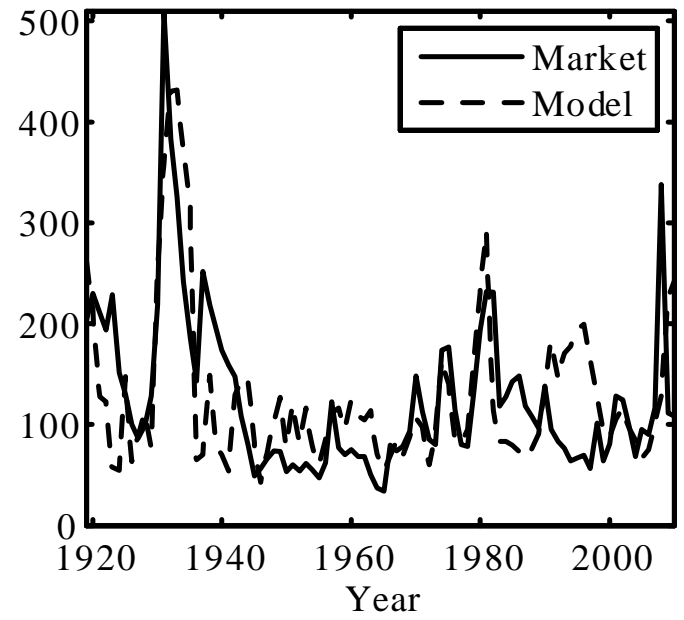

Notes to Figure: We obtain the innovation of the historical log consumption growth rates from the St. Louis Fed and Robert Shiller's website. We use this innovation to construct the time series of the surplus ratio and loss rate using (1)-(2) and (13), respectively. Given the time series of $S$ and $L$, we back out the model implied spreads and compare them to historical data. Each panel shows a different version of the model. The full model is in the bottom right panel. 
Figure 6. The Impact of Idiosyncratic Default
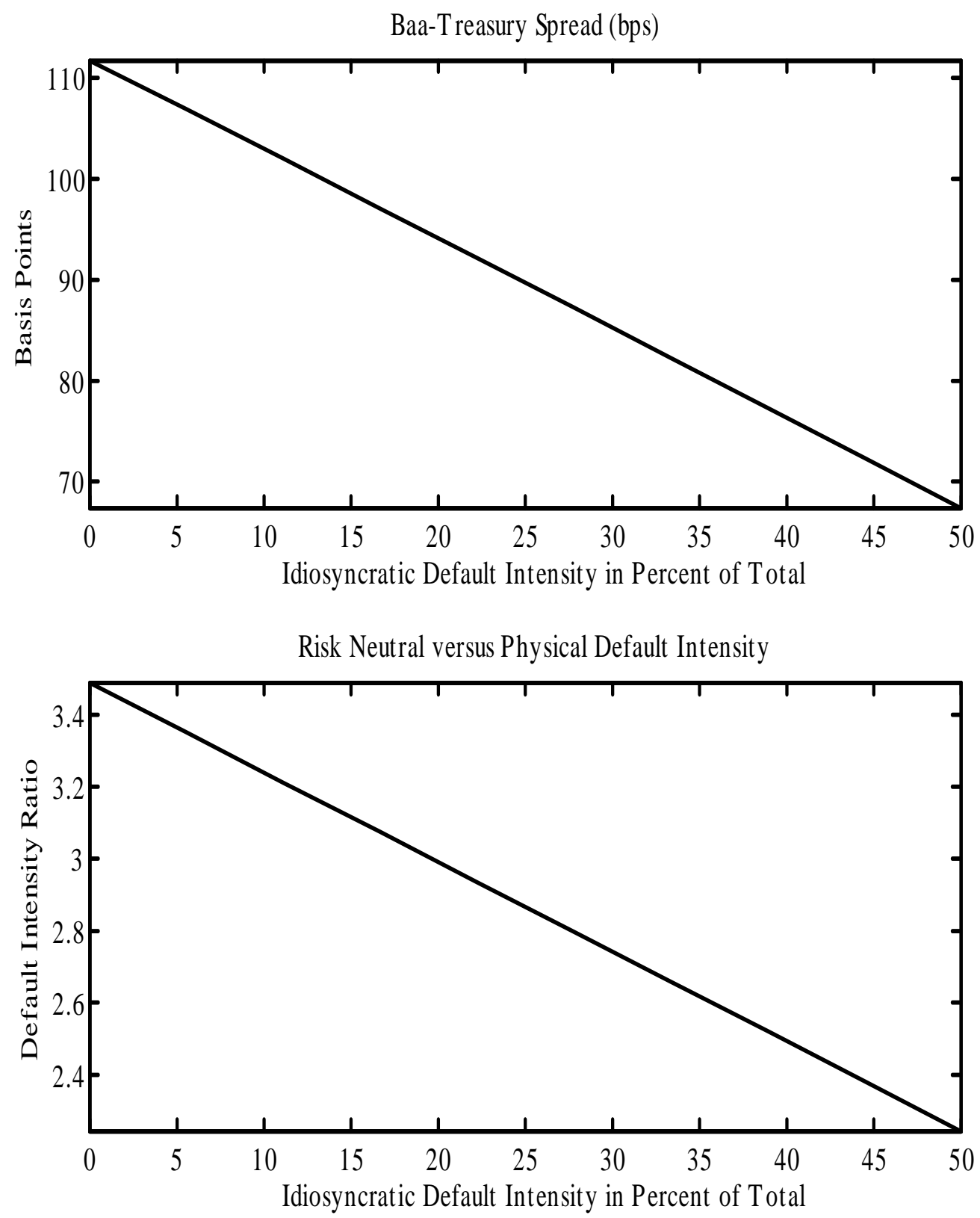

Notes to Figure: The top panel plots the 5-year Baa-Treasury model-implied spread as a function of the idiosyncratic default intensity in percent of the total default intensity, $\lambda^{\text {idio }} /\left(\lambda^{\text {sys }}+\lambda^{\text {idio }}\right)$. The bottom panel shows the model-implied ratio of default intensity between $P$ and $Q$ measures, $\lambda_{t}^{Q} / \lambda$, plotted against the idiosyncratic default intensity in percent of the total. 
Figure 7. Model-Implied Spread Sensitivities
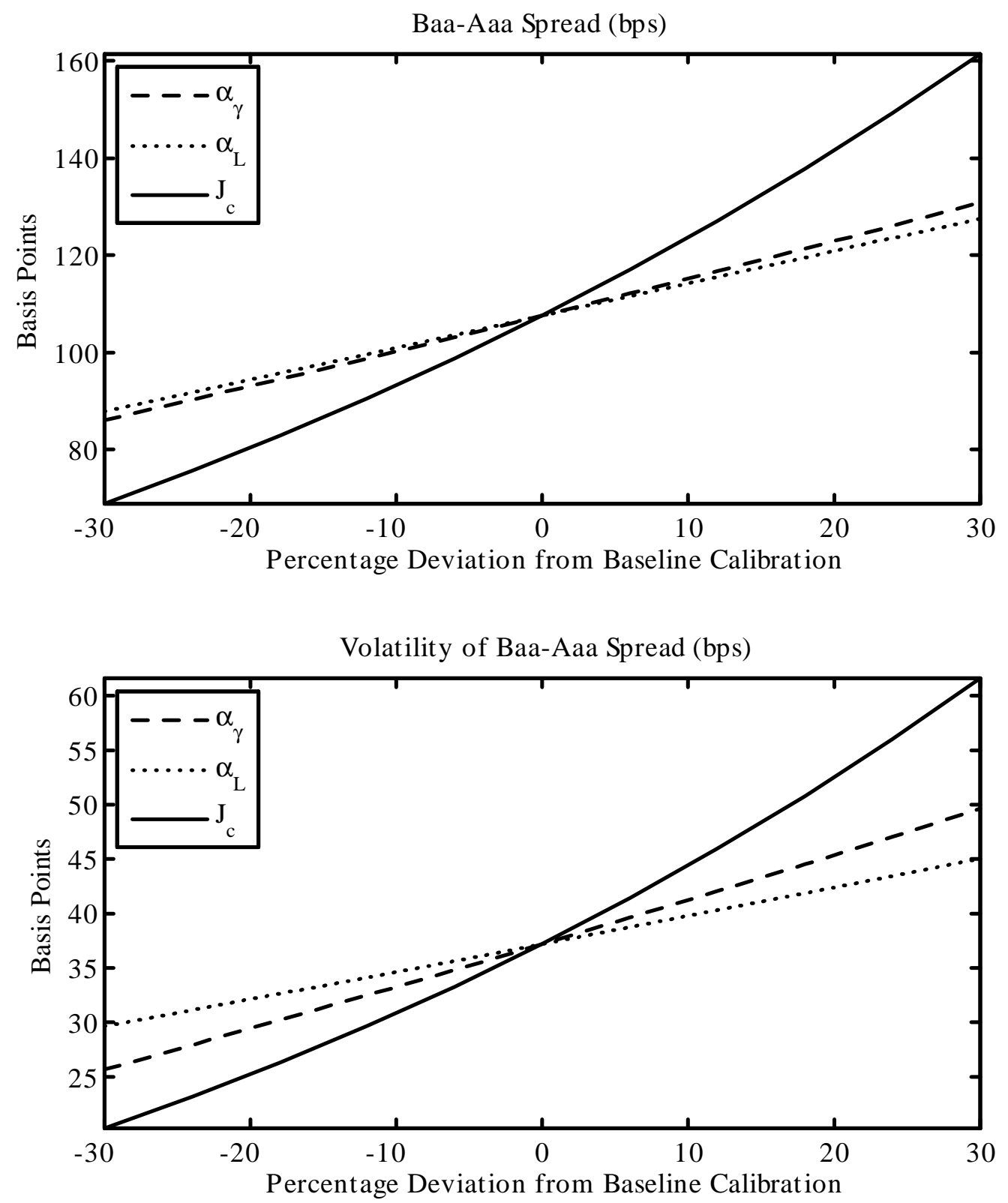

Notes to Figure: The top panel plots the sensitivity of the average model-implied 5-year Baa-Aaa spread level to changes in three key parameters: The sensitivity of habit and loss to consumption shocks $\left(\alpha_{\gamma}\right.$ and $\left.\alpha_{L}\right)$, and the absolute consumption jump size $\left(\left|J_{c}\right|\right)$. The bottom panel plots the sensitivity of the volatility of the 5-year model-implied Baa-Aaa spreads to changes in the same three parameters. 
Figure 8. Baa-Aaa Spreads, Option Skewness and Consumption Jumps Size
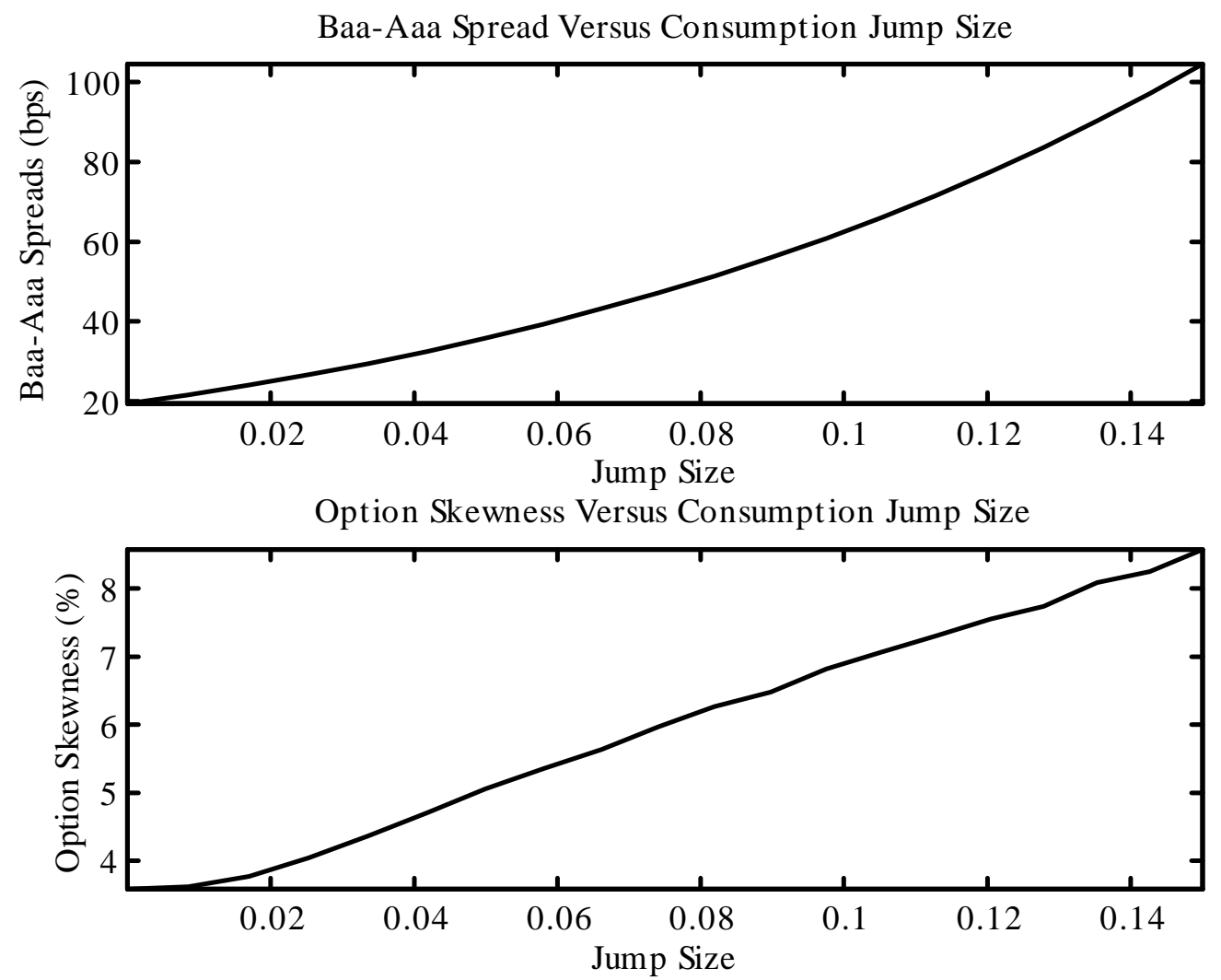

Baa-Aaa Spread Versus Option Skewness

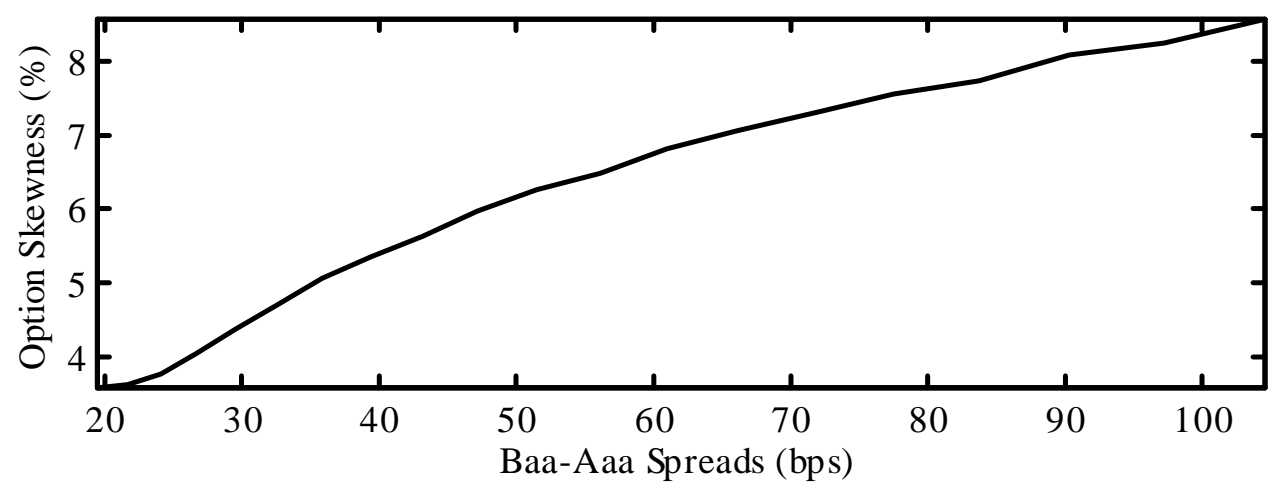

Notes to Figure: The top panel shows the model-implied Baa-Aaa spread as the consumption disaster magnitude, $J_{C}$ varies from zero to 0.15 which is the benchmark calibration value. The middle panel plots option skewness (the implied volatility difference between 10\% OTM puts and ATMs) against $J_{C}$. In the bottom panel we plot option skewness in the middle panel against the Baa-Aaa spreads from the top panel. 
Figure 9. Model-Implied Option Skewness and Credit Spreads across the State of the Economy
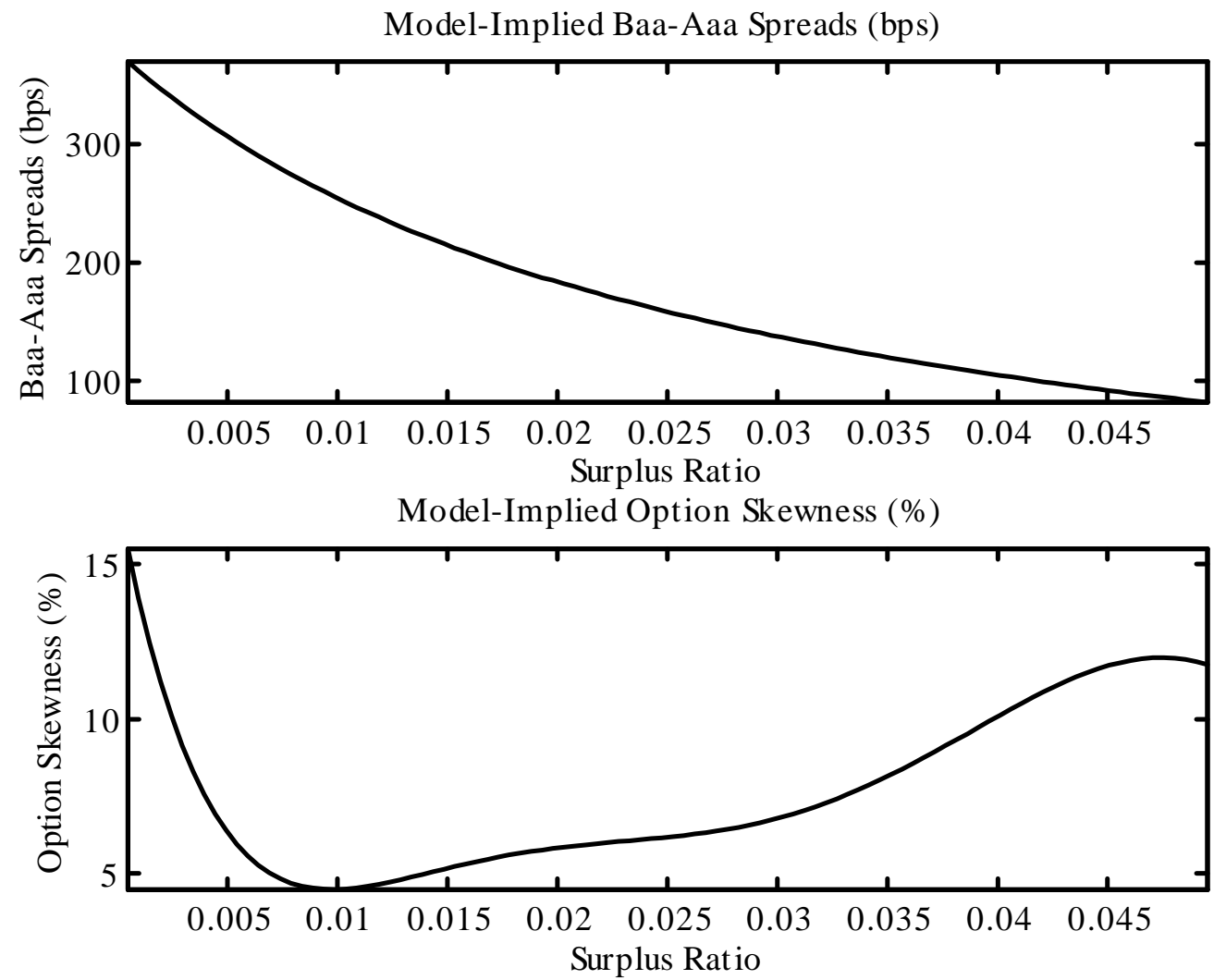

Model-Implied Option Skewness (\%) versus Model-Implied Baa-Aaa Spreads (bps)

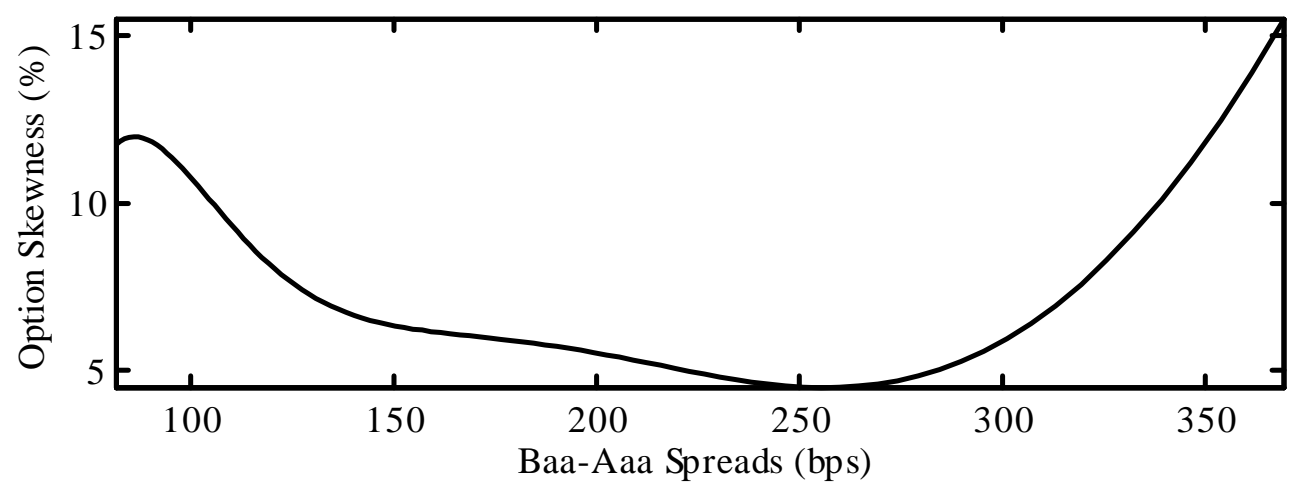

Notes to Figure: The top panel plots the model-implied 5-year Baa-Aaa spread against the surplus ratio. The middle panel plots option skewness against the surplus ratio. Option skewness is measured as the implied volatility difference between 10\% OTM puts and ATMs. The bottom panel plots the credit spread from the top panel against option skewness from the middle panel. 
2013-27: $\quad$ Nima Nonejad: Particle Markov Chain Monte Carlo Techniques of Unobserved Compdonent Time Series Models Using Ox

2013-28: $\quad$ Ulrich Hounyo, Sílvia Goncalves and Nour Meddahi: Bootstrapping preaveraged realized volatility under market microstructure noise

2013-29: Jiti Gao, Shin Kanaya, Degui Li and Dag Tjøstheim: Uniform Consistency for Nonparametric Estimators in Null Recurrent Time Series

2013-30: Ulrich Hounyo: Bootstrapping realized volatility and realized beta under a local Gaussianity assumption

2013-31: Nektarios Aslanidis, Charlotte Christiansen and Christos S. Savva: Risk-Return Trade-Off for European Stock Markets

2013-32: Emilio Zanetti Chini: Generalizing smooth transition autoregressions

2013-33: Mark Podolskij and Nakahiro Yoshida: Edgeworth expansion for functionals of continuous diffusion processes

2013-34: Tommaso Proietti and Alessandra Luati: The Exponential Model for the Spectrum of a Time Series: Extensions and Applications

2013-35: Bent Jesper Christensen, Robinson Kruse and Philipp Sibbertsen: A unified framework for testing in the linear regression model under unknown order of fractional integration

2013-36: Niels S. Hansen and Asger Lunde: Analyzing Oil Futures with a Dynamic Nelson-Siegel Model

2013-37: Charlotte Christiansen: Classifying Returns as Extreme: European Stock and Bond Markets

2013-38: $\quad$ Christian Bender, Mikko S. Pakkanen and Hasanjan Sayit: Sticky continuous processes have consistent price systems

2013-39: Juan Carlos Parra-Alvarez: A comparison of numerical methods for the solution of continuous-time DSGE models

2013-40: $\quad$ Daniel Ventosa-Santaulària and Carlos Vladimir Rodríguez-Caballero: Polynomial Regressions and Nonsense Inference

2013-41: Diego Amaya, Peter Christoffersen, Kris Jacobs and Aurelio Vasquez: Does Realized Skewness Predict the Cross-Section of Equity Returns?

2013-42: Torben G. Andersen and Oleg Bondarenko: Reflecting on the VPN Dispute

2013-43: $\quad$ Torben G. Andersen and Oleg Bondarenko: Assessing Measures of Order Flow Toxicity via Perfect Trade Classification

2013-44: $\quad$ Federico Carlini and Paolo Santucci de Magistris: On the identification of fractionally cointegrated VAR models with the $F(d)$ condition

2013-45: $\quad$ Peter Christoffersen, Du Du and Redouane Elkamhi: Rare Disasters and Credit Market Puzzles 